\title{
DEVELOPMENTS IN SCHAUDER BASIS THEORY ${ }^{1}$
}

\author{
BY C. W. McARTHUR ${ }^{2}$
}

1. Introduction. In the forty-four years since 1927 when J. Schauder [101] introduced the notion of a topological basis for a Banach space, well over two hundred papers on basis theory have been published. About one-fifth of these appeared in the twenty-three years before 1950 with the other four-fifths appearing in the twenty-one years from 1950 to the present. Surely the devastating effect of World War II is one factor in this distribution of mathematical output. Indeed, two of the originators of the abstract theory of bases, J. Schauder and S. Banach, were essentially casualties of the war, Schauder losing his life during the war and Banach dying prematurely shortly thereafter.

It will be the purpose of this paper to trace the development up to the present time of several theorems or problems introduced in 1932 by Banach [6], as well as to report on some developments not anticipated by Banach.

We first mention a problem considered by Fréchet and Banach which has only recently been solved and in whose solution basis theory played an important role. Which of the topological linear spaces are homeomorphic? In 1928, [33, pp. 94-96] Fréchet asked whether the separable Hilbert space $l^{2}$ was homeomorphic to $(s)$, the space of all real sequences with the product topology. In 1932, [6, p. 233] Banach stated that Mazur had shown that $(s)$ was not homeomorphic to $l^{2}$. It was subsequently realized that the question was still open. Indeed, in 1966 [1], R. D. Anderson was the first to prove that $l^{2}$ and (s) are homeomorphic. C. Bessaga and A. Pelczynski [8], [13] in the meantime had shown that "Under the conjecture that all separable infinite dimensional Banach spaces are homeomorphic to $l^{2}$, every separable infinite dimensional Fréchet space $E$, with $E \neq(s)$ is homeomorphic to $l^{2}$." Thus when M. I. Kadec [53] showed, using basis theory as a main tool, that all separable infinite dimensional Banach spaces are homeomorphic, Anderson's result provided the missing link to give the startling result: All separable infinite dimensional Fréchet spaces are homeomorphic.

\footnotetext{
${ }^{1}$ An expanded version of an invited address delivered to the 689th meeting of the Society in Auburn, Alabama on November 20,1971; received by the editors January 10, 1972.

AMS 1970 subject classifications. Primary 46A35, 46A40, 46A45, 46B15; Secondary 46-02.

Key words and phrases. Schauder basis, bounded approximation property, biorthogonal system, basis cone, weak basis, shrinking basis, boundedly complete basis, conditional basis, block basis, universal basis, Markushevich basis, selection principle, basic sequence, Dragilev-space, Choquet-simplex.

2 The research for this paper was supported in part by NSF grant GP-9632.
} 
Throughout this lecture $E$ will denote a Hausdorff topological vector space or some specialization thereof and $E^{\prime}$ the space of continuous linear functionals on $E$. A pair of sequences $\left\{x_{i}\right\} \subset E$ and $\left\{f_{i}\right\} \subset E^{\prime}$ is a biorthogonal system for $E$ provided that $f_{i}\left(x_{j}\right)=\delta_{i j}$. S. Banach [6] devoted a chapter of his book to biorthogonal systems. A biorthogonal system $\left\{x_{i}, f_{i}\right\}$ is a Schauder basis for $E$ if and only if the series $\sum_{i=j}^{\infty} f_{i}(x) x_{i}$ converges to $x$ for each $x \in E$. In 1927, J. Schauder introduced the concept of Schauder basis for Banach spaces and constructed a Schauder basis for $C[0,1]$. Note that if $\left\{x_{i}, f_{i}\right\}$ is a Schauder basis for $E$ and $x=\sum_{i=1}^{\infty} a_{i} x_{i}$ then $a_{i}=f_{i}(x), i=1,2, \ldots$. Thus the coefficients in the expansion of an element in a Schauder basis are unique. A sequence $\left\{x_{i}\right\}$ in $E$ is a (topological) basis for $E$ if and only if corresponding to each $x \in E$ there is a unique sequence of scalars $\left\{a_{i}\right\}$ such that the series $\sum_{i=1}^{\infty} a_{i} x_{i}$ converges to $x^{3}$ A sequence $\left\{x_{i}\right\}$ in $E$ is basic iff it is a basis for its closed linear span $\left[x_{i}\right]$. When $\left\{x_{i}\right\}$ is a basis for $E$ we define the coefficient functionals $f_{i}$ on $E$ by $f_{i}(x)=a_{i}$ if $x=\sum_{j=1}^{\infty} a_{j} x_{j}, x \in E, i=1,2, \ldots$. Banach [6, p. 111] showed that the coefficient functionals of a basis for a Banach space are necessarily continuous, i.e., a basis for a Banach space is a Schauder basis.

The literature on bases now contains two books [73], [112] which have been published in the last three years.

2. Bases and the approximation property. The question, "Does every separable Banach space have a basis" was posed by Banach [6, pp. 111$112,245]$. It has remained unanswered for thirty-nine years and is now known as "the basis problem." Recent work reveals that the basis problem is closely related to another important problem of functional analysis -."the approximation problem."

A Banach space $E$ is said to have the approximation property iff corresponding to each compact set $K \subset E$ and $\varepsilon>0$ there exists a finite dimensional bounded linear operator $F$ from $E$ into $E$ such that $\|x-F(x)\|<\varepsilon$ for all $x \in K$. If, moreover, there exists a constant $\lambda>0$ which is independent of $K$ and $\varepsilon$ and such that $F$ can be chosen with $\|F\| \leqq \lambda$ then $E$ is said to have the bounded approximation property. A Banach space with a basis has the approximation property $\left[112\right.$, p. 170] ${ }^{4}$

Grothendieck [40] proved that if $E$ is reflexive or $E$ is a separable dual space, then the approximation property for $E$ implies the bounded approximation property for $E$ implies the bounded approximation property

\footnotetext{
${ }^{3}$ If the basis expansion for each $x$ in $E$ converges unconditionally the basis is an unconditional basis. In the contrary case, the basis is a conditional basis.

${ }^{4}$ In June 1972 Per Enflo, at an analysis conference at Hebrew University, Jerusalem, Israel, announced the result: There exists a Banach space without the approximation property and thus there exists a separable Banach space without a basis.
} 
with $\lambda=1$. It would be very desirable to have a simple proof of Grothendieck's result proved without using his machinery of tensor products.

In order to make a first connection between the basis problem and the approximation problem, we state a recent important result on bases. S. Karlin [58] in 1948 asked if a sufficient condition for a Banach space $E$ to have a basis would not be for $E^{\prime}$ to have a basis in its strong topology. That this condition is indeed sufficient has just been shown by Johnson, Rosenthal, and Zippin [51]. They have, in fact, shown more. A basis $\left\{x_{i}\right\}$ for a Banach space is shrinking iff for each $f \in E^{\prime}$, $\lim _{n \rightarrow \infty} \sup \left\{|f(x)|: x \in\left[x_{k}\right]_{k=n}^{\infty},\|x\| \leqq 1\right\}=0$. A shrinking basis $\left\{x_{i}, f_{i}\right\}$ for a Banach space has the property that $\left\{f_{i}\right\}$ is a basis for $E^{\prime}$ with its strong topology. Their theorem is:

THEOREM 2.1. Let $E$ be a Banach space. Then $E$ has a shrinking basis if either of the following holds:

(a) E' has a basis;

(b) E has a basis and $E^{\prime}$ is (strongly) separable and has the bounded approximation property.

J. Lindenstrauss [65] improved a theorem of James [47] to the following:

THEOREM 2.2. For any separable Banach space E there exists a Banach space $Y$ with a monotone shrinking basis such that

(1) $E$ is a quotient of $Y^{\prime}$, and

(2) $Y^{\prime \prime}=J Y \oplus E^{\prime}$,

where $J(Y)$ is the canonical embedding of $Y$ into $Y^{\prime \prime}$ and $J(Y) \oplus E^{\prime}$ is the direct sum of $J(Y)$ and $E^{\prime}$. ( $A$ basis $\left\{\psi_{n}\right\}$ is monotone iff $\left\|\sum_{i=1}^{n} a_{i} \psi_{i}\right\|$ $\leqq\left\|\sum_{i=1}^{n+1} a_{i} \psi_{i}\right\|$ for arbitrary scalars $a_{1}, a_{2}, \ldots, a_{n+1}$.)

Lindenstrauss [65] obtains the following corollary.

COROLlaRY 2.3. Either every Banach space $E$ has the approximation property or there is a Banach space $W$ such that

(a) $W$ has a basis, and

(b) $W^{\prime}$ is separable but does not have the approximation property and hence does not have a basis.

Proof. It follows from the definition and the Hahn-Banach theorem that if there is a Banach space which does not have the approximation property, then there is a separable space $E$ which fails to have the approximation property. As shown by Grothendieck [40] we may assume that $E$ is a subspace of $c_{0}$. Letting $W=Y^{\prime}$ where $Y$ is defined by Theorem 2.2, we have that $W$ has a basis and $W^{\prime}$ is separable. By theorem 2.2, $W=Y^{\prime}$ has a basis. By part (2) of the theorem $E^{\prime}$ is isomorphic to a complemented 
subspace of $W^{\prime}$ and since $E^{\prime}$ does not have the approximation property, it follows that $W^{\prime}$ does not have the approximation property. We use here a result of Grothendieck [40]; namely, if $E^{\prime}$ has the approximation property so does $E$.

Finally, Johnson, Rosenthal, and Zippin [51] and independently Pelczynski [91] proved

THEOREM 2.4. A separable Banach space has the bounded approximation property iff it is isomorphic to a complemented subspace of a Banach space with a basis.

A weaker version of Theorem 2.4 with finite dimensional Schauder decomposition instead of basis was proved earlier by Pelczynski and Wojtaszczyk [94]. In the above mentioned paper of Lindenstrauss, Theorem 2.4 was proved with the additional assumption that the space is a separable dual. The proof of Johnson-Rosenthal-Zippin is based on Theorems 2.1 and 2.2 above and the construction of Pelczynski and Wojtaszczyk mentioned above. The proof of Pelczynski [91] is direct (uses only the Auerbach lemma) and can be generalized to Fréchet spaces.

W. Johnson has established strong connections between Markushevich bases (biorthogonal systems $\left\{x_{n}, f_{n}\right\}$ with $\left\{x_{n}\right\}$ fundamental and $\left\{f_{n}\right\}$ total) and approximation properties. He has shown $[\mathbf{4 8}]$ that a separable Banach $E$ space has the bounded approximation property iff $E$ admits a Markuschevich basis which is a generalized summation basis in the sense of Kadec [48]. Johnson [49] also shows the following: Let $E$ be a separable complex Banach space. If $E^{\prime}$ has the bounded approximation property, then there exists a Markushevich basis $\left\{x_{n}, f_{n}\right\}$ for $E$ and a set $\left\{\lambda_{i, n}: i=1,2, \ldots, n ; n=1,2, \ldots\right\}$ of scalars such that for each $x \in E$, $x=\lim _{n \rightarrow \infty} \sum_{i=1}^{n} \lambda_{i, n} f_{i}(x) x_{i}$.

3. The existence of basic sequences. If $E$ is a topological vector space from what subsets of $E$ can one select a basic sequence? A first answer to this question was given by Banach [6, p. 238] who stated without proof that every infinite dimensional Banach space $E$ contains an infinite dimensional closed subspace with a basis. Different proofs of this were given in 1958 by Gelbaum [39] and by Bessaga and Pelczynski [9], and in 1962 by Day [21], [22]. Day, in fact, proved that in every infinite dimensional normed space $E$ there exists a biorthogonal system $\left\{x_{n}\right\} \subset E$, $\left\{f_{n}\right\} \subset E^{\prime}$ such that $\left\{x_{n}\right\}$ is basic, $\left\|x_{n}\right\|=\left\|f_{n}\right\|=1, n=1,2, \ldots$, and the norm of the $m$ th partial sum operator is no greater than $1+1 / m$. Bessaga and Pelczynski [9] state the following theorem.

THEOREM 3.1. Let $\left\{x_{n}, f_{n}\right\}$ be a basis for a complete metric linear space $E$ which either is locally convex (i.e., is a Fréchet space) or else has a bounded neighborhood of zero. If a sequence $\left\{y_{n}\right\}$ in $E$ satisfies the conditions 
$\lim _{n \rightarrow \infty} f_{i}\left(y_{n}\right)=0, i=1,2, \ldots$, and there exists a neighborhood $V$ of 0 such that $y_{n} \notin V, n=1,2, \ldots$, then there exists a subsequence $\left\{y_{p_{n}}\right\}$ which is a basic sequence equivalent to a block basis. (Definition, §7.)

COROLlaRY 3.2. (a) Each infinite dimensional Fréchet space contains a basic sequence.

(b) If $Y$ is an infinite dimensional subspace of a complete metric linear space $E$ with a bounded neighborhood of zero and a basis, then $Y$ contains a basic sequence.

In a recent paper of Joel $\mathrm{H}$. Shapiro [103] a selection principle for another class of nonlocally convex spaces is developed. If $E$ is a real or complex linear space, a nonnegative, subadditive functional $\|\cdot\|$ is called an $F$-norm if it has the additional properties:

(a) $\|x\|=0$ iff $x=\theta$.

(b) $\|t x\| \leqq\|x\|$ for all scalars $t$ with $|t| \leqq 1$.

(c) $\lim _{n \rightarrow \infty}\|(1 / n) x\|=0$ for each $x \in E$.

(d) The metric $d(x, y)=\|x-y\|$ is complete.

A linear space equipped with an $F$-norm is an $F$-space. A nonnegative, subadditive functional $S$ on a linear space $E$ is called a p-seminorm $(0<p \leqq 1)$ iff $S(t x)=|t|^{p} S(x)$ for all scalars $t$ and vectors $x$, and a $p$-norm iff, in addition, $x=\theta$ whenever $S(x)=0$. If the topology of $E$ is induced by a family $\left\{S_{i}\right\}$ of $p_{i}$-seminorms, then $E$ is called locally pseudo-convex (or semi-convex). Shapiro's selection principle is

THEOREM 3.3. Let $E$ be a locally pseudo-convex $F$-space with a basis $\left\{x_{n}, f_{n}\right\}$. If $\left\{y_{n}\right\}$ is a sequence in $E$ with $\inf \left\|y_{n}\right\|>0$ and $\lim _{n} f_{i}\left(y_{n}\right)=0$, $i=1,2, \ldots$, then there exists a basic subsequence $\left\{y_{n_{k}}\right\}$ equivalent to a block basis with respect to $\left\{x_{n}\right\}$.

Shapiro uses the above theorem in giving an example of a nonlocally convex $F$-space with the property that every infinite dimensional subspace contains a further infinite dimensional locally convex subspace.

M. I. Kadec and Pelczynski [55] have given further criteria for the existence of basic sequences.

Let $E$ be a Fréchet space and $E^{\prime}$ the conjugate space to $E$. A set $\Gamma \subset E^{\prime}$ is said to be norming for $E$ iff for every fundamental system of bounded sets $\left\{B_{n}\right\}$ in $E^{\prime}$ the sequence $\left\{\|\cdot\|_{B_{n} \cap \Gamma}\right\}$ with $\|x\|_{B_{n} \cap \Gamma}=\sup \left\{|f(x)|: f \in B_{n} \cap \Gamma\right\}$ for $x \in E$ is a generating family of seminorms for the topology of $E$. The set $\Gamma=E^{\prime}$ is an example of a norming set. We shall write $x_{n} \rightarrow_{\Gamma} 0$ iff $f\left(x_{n}\right) \rightarrow 0$ for every $f \in \Gamma$.

The next three theorems are the main results of Kadec and Pelczynski $[55]$ on the selection of basic sequences.

THEOREM 3.4. $A$ subset $M$ in an arbitrary Fréchet space $E$ contains a basic 
sequence iff there is a norming set $\Gamma \subset E^{\prime}$ and a sequence $\left\{x_{n}\right\}$ of elements of $M$ such that $x_{n} \neq 0(n=1,2, \ldots)$ and $t_{n} x_{n} \rightarrow_{\Gamma} 0$ for every sequence of scalars $\left\{t_{n}\right\}$.

THEOREM 3.5. Let $\left\{x_{n}\right\}$ be a sequence in a Fréchet space $E$ which does not converge to zero and suppose $x_{n} \rightarrow_{\Gamma} 0$ for some norming subset $\Gamma \subset E^{\prime}$. Then $\left\{x_{n}\right\}$ contains a basic sequence.

THEOREM 3.6. Let $M$ be a bounded noncompact set in a Fréchet space E. Then there exists a basic sequence in $M$.

V. D. Milman and J. B. Tumarkin [82] point out that Theorem 3.5 above in the case $\Gamma=E^{\prime}$ has a hypothesis which is never satisfied in a nuclear or, indeed, a Montel space. They offer the following theorem "free of this defect."

THEOREM 3.7. Let $E$ be a Fréchet space or an $(L F)$ space (a certain countable inductive limit of Fréchet spaces). If $\left\{x_{n}\right\}_{n=1}^{\infty}$ is a sequence in $E$ such that for every sequence $\left\{c_{k}\right\}\left(c_{k}>0\right),\left\{c_{k} x_{k}\right\}_{k=1}^{\infty}$ has no subsequence weakly converging to $x_{0} \neq 0$, then $\left\{x_{n}\right\}$ contains a basic subsequence.

In 1962 V. I. Gurarii [41] showed that in every infinite dimensional Banach space there exists a basic sequence which is a conditional basis for its closed linear span. It is not known whether every infinite dimensional Banach space contains an infinite dimensional subspace with an unconditional basis. However, it has been shown [12] that in each Fréchet space which is not isomorphic to any Banach space there is an (infinite) unconditional basic sequence (spanning a nuclear space).

Call a biorthogonal system $\left\{x_{n}, f_{n}\right\}$ bibasic if $\left\{x_{n}\right\}$ is a basic sequence in $E$ and $\left\{f_{n}\right\}$ is basic in the strong topology of $E^{\prime}$. It can be shown that if $E$ is locally convex and the partial sum operators $s_{n}(x)=\sum_{i=1}^{n} f_{i}(x) x_{i}$, $n=1,2, \ldots, x \in E$, are equicontinuous then $\left\{x_{i}, f_{i}\right\}$ is bibasic. In particular a Schauder basis $\left\{x_{i}, f_{i}\right\}$ for a Fréchet space is bibasic. However, an arbitrary biorthogonal system $\left\{x_{i}, f_{i}\right\}$ in a Banach space with $\left\{x_{i}\right\}$ basic need not be bibasic. It has recently been shown by Davis, Dean, and Lin [18] that every infinite dimensional Banach space $E$ admits a biorthogonal system $\left\{x_{i}, f_{i}\right\},\left\{x_{i}\right\} \subset E,\left\{f_{i}\right\} \subset E^{\prime}$, which is bibasic.

Johnson and Rosenthal [50] make the following definitions. Let $E$ be a Banach space. A sequence $\left\{f_{n}\right\} \subset E^{\prime}$ is called $w^{*}$-basic provided that there is a sequence $\left\{x_{n}\right\} \subset E$ so that $\left\{x_{n}, f_{n}\right\}$ is biorthogonal and the series $\sum_{i=1}^{\infty} f\left(x_{i}\right) f_{i}$ is $w^{*}$ convergent to $f$ for each $f$ in the $w^{*}$ closure of $\left[f_{n}\right]$. Johnson and Rosenthal [50] prove the following $w^{*}$-analogue of the Bessaga-Pelczynski selection principle.

TheOREM 3.8. Suppose that $E$ is a separable Banach space, $\left\{f_{n}\right\} \subset E^{\prime}$, $f_{n} \stackrel{w^{*}}{\rightarrow} 0$, and $\lim \sup \left\|f_{n}\right\|>0$. Then $\left\{f_{n}\right\}$ has a $w^{*}$-basic subsequence, $\left\{f_{n_{i}}\right\}$. 
Furthermore, $\left\{f_{n_{i}}\right\}$ may be chosen such that if $\left\{z_{n_{i}}\right\} \subset E$ is selected with $\left\{z_{n_{i}}, f_{n_{i}}\right\}$ biorthogonal and $\left.\left.s_{m}: \tilde{[} f_{n_{i}}\right] \rightarrow \tilde{[} f_{n_{i}}\right]$ is defined $($ for $m=1,2, \ldots)$ by $s_{m}(f)=\sum_{i=1}^{m} f\left(z_{n_{i}}\right) f_{n_{i}}$ for all $f \in\left[\tilde{[} f_{n_{i}}\right]$, then $\left\|s_{m}\right\| \rightarrow 1$. $(\tilde{A}$ denotes the $w^{*}$ closure of $A$.)

They use this as a main lemma for the following results which solve two problems posed by Pelczynski [89].

(a) If $E$ is a separable infinite dimensional Banach space, then $E$ has a quotient space with a basis, i.e., there exists a closed subspace $M$ of infinite codimension in $E$ such that $E / M$ has a basis.

(b) Every separable conjugate space contains a boundedly complete basic sequence.

It was shown by Markushevich [72] that every separable Banach space admits a Markushevich basis. Banach [6] asked, "Does every separable Banach space admit a bounded Markushevich basis?" i.e., a Markushevich basis $\left\{x_{n}, f_{n}\right\}$ with $\sup \left\{\left\|x_{n}\right\|\left\|f_{n}\right\|: n \in \omega\right\}<+\infty$. This question remains open. However, Davis and Johnson have shown [20] that if $E$ is a separable Banach space, then to each $\varepsilon>0$ corresponds a biorthogonal system $\left\{x_{n}, f_{n}\right\}$ with $\sup _{n}\left\|x_{n}\right\|\left\|f_{n}\right\|<1+\varepsilon$ which may be selected either so that $\left\{x_{n}\right\}$ is fundamental or $\left\{f_{n}\right\}$ is total.

Let $\left\{x_{n}, f_{n}\right\}$ be a biothogonal system with $\left\{f_{n}\right\}$ total for a Banach space $E$. A scalar sequence $\left\{a_{n}\right\}$ is a multiplier of an element $x$ in $E$ with respect to $\left\{x_{n}, f_{n}\right\}$ iff there exists $y$ in $E$ with $f_{n}(y)=a_{n} f_{n}(x), n=1,2, \ldots$. Let $M\left(x,\left\{x_{n}, f_{n}\right\}\right)$ denote the set of all multipliers of $x$ and let $M\left(E,\left\{x_{n}, f_{n}\right\}\right)$ $=\bigcap\left\{M\left(x,\left\{x_{n}, f_{n}\right\}\right): x \in E\right\}$.

Mityagin [84] raised the question: If $E$ is separable and $M\left(E,\left\{x_{n}, f_{n}\right\}\right)$ contains every sequence of the form $\left\{\varepsilon_{n}\right\}$ where $\varepsilon_{n}$ is either 0 or 1 for each $n$, is $\left\{x_{n}\right\}$ an unconditional basis for $E$ ?

Kadec and Pelczynski [55] subsequently raised this question: If $E$ is separable and $x$ is an element of $E$ such that $M\left(x,\left\{x_{n}, f_{n}\right\}\right)$ contains each sequence of the form $\left\{\varepsilon_{n}\right\}$ with $\varepsilon_{n}$ equal to either 0 or 1 for each $n$, does the series $\sum_{n=1}^{\infty} f_{n}(x) x_{n}$ converge unconditionally to $x$ ?

An affirmative answer to the second question clearly answers the first affirmatively. The first question was settled affirmatively by Davis, Dean, and Singer [19] and the second one was answered affirmatively by Bachelis and Rosenthal [5] for Banach spaces which do not contain a subspace isomorphic to $(m)$ and, in particular, for separable Banach spaces.

4. The continuity and weak basis theorems. Although Banach gave a proof only for Banach spaces [6], he appears to have known this more general theorem.

TheOREM 4.1 (CONTINUITY TheOREM). A basis for a complete metric linear space is a Schauder basis. 
He appears to implicity use the above theorem in proving that the separable complete metric linear space $S[6$, p. $9 ;$ p. 239] does not admit a basis since $S$ has no nontrivial continuous linear functionals. Recall that $S$ is the space of Lebesgue measurable functions on the interval $[0,1]$ with the distance between $x$ and y defined by $\int_{0}^{1}|x(t)-y(t)| /(1+|x(t)-y(t)|) d t$.

Proofs of Theorem 4.1 have been given by Newns [86] for the Fréchet space case and independently by Nikolskii [87], Bessaga and Pelczynski [11], and Arsove [2] for the complete metric linear case. All of these proofs, like Banach's, depend upon the open mapping or closed graph theorems. Does the continuity theorem really depend upon the open mapping theorem? It is known that a $w^{*}$-basis for the dual of a Banach space need not be a $w^{*}$-Schauder basis. The $w^{*}$-topology is not barrelled. Could it be that a basis for a sequentially complete barrelled space is a Schauder basis?

We next consider the weak basis theorem.

THEOREM 4.2. A weak basis for a Fréchet space $E$ (i.e., a basis in the weak topology of $E$ ) is a basis for $E$ with its initial topology.

The above theorem is stated for the Banach space case in the appendix of Banach's monograph [6, p. 238] and is attributed to S. Mazur by his students. Karlin [58] was the first to sketch a proof of the weak basis theorem for Banach spaces. Day [21] gave proofs of the theorem with the additional assumption that either the space was weakly sequentially complete or the coefficient functionals were continuous. Bessaga and Pelczynski [11] were the first to prove that a weak basis for a Fréchet space is a basis. Ruckle [96] proved that a weak basis of closed subspaces of a Banach space is a basis of subspaces. (A sequence of subspaces $\left\{M_{i}\right\}$ of $E$ is a basis of subspaces of $E$ if corresponding to each $x \in E$ there exists a unique sequence $\left\{x_{i}\right\}, x_{i} \in M_{i}$, such that $x=\sum_{i=1}^{\infty} x_{i}$.

Arsove and Edwards [3] showed that

THEOREM 4.3. A weak Schauder basis for a barrelled space $E$ is a Schauder basis for $E$.

This theorem is a corollary of an earlier result of Dieudonné [25] which says, in effect, that a sufficient condition for a biorthogonal system $\left\{x_{i}\right\} \subset E,\left\{f_{i}\right\} \subset E^{\prime}$, to be bibasic where $E$ is a barrelled space is that $\left\{\sum_{i=1}^{n} f_{i}(x) x_{i}\right\}_{n=1}^{\infty}$ be bounded for each $x \in E$. This boundedness condition is clearly satisfied when $\left\{x_{i}, f_{i}\right\}$ is a weak Schauder basis. Furthermore, $E=\left[x_{i}\right]$ when $\left\{x_{i}, f_{i}\right\}$ is a weak Schauder basis since the weak closure and the closure of a subspace of a locally convex space coincide.

A relatively simple proof of the weak basis theorem was discovered by the author [75] who actually proved the following slight generalization. 
THEOREM 4.4. $A$ weak basis of closed subspaces of a Fréchet space is a Schauder basis of subspaces of $E$. ( $A$ basis of subspaces $\left\{M_{i}\right\}$ of $E$ is a Schauder basis of subspaces if the projection $P_{n}(x)=x_{n}$ where $x=\sum_{i=1}^{\infty} x_{i}$, $x_{i} \in M_{i}, i=1,2, \ldots$, is continuous on $E$ for each $n$.)

We will sketch a proof of Theorem 4.4 in order to make explicit a principle used implicitly in the proof [75]. The principle is stated in the following lemma which can be used for a number of purposes in basis theory [76].

LEMma. Let $\left\{S_{i}\right\}_{i \in I}$ be a family of linear functions from a vector space $E$ into a linear topological space $\{F, \mathscr{T}\}$ and suppose $\left\{S_{i}(x): i \in I\right\}$ is bounded for each $x \in E$. Then there exists a weakest vector topology $\mathscr{T}^{\prime}$ for $E$ such that $\left\{S_{i}\right\}_{i \in I}$ is $\mathscr{T}^{\prime}-\mathscr{T}$ equicontinuous. If $V=\{V\}$ is any local base of balanced $\mathscr{T}$-neighborhoods of $\theta$ for $(F, \mathscr{T})$ then $\mathscr{V}^{\prime}$ $=\left\{V^{\prime}: V \in \mathscr{V}\right\}$ where $V^{\prime}=\bigcap_{i \in I} S_{i}^{-1}(V)$ is a local base of balanced neighborhoods of $\theta$ for $\left(E, \mathscr{T}^{\prime}\right)$. If $(F, \mathscr{T})$ is locally convex so is $\left(E, \mathscr{T}^{\prime}\right)$. If $(F, \mathscr{T})$ is pseudo metrizable so is $\left(E, \mathscr{T}^{\prime}\right)$. If $(F, \mathscr{T})$ is Hausdorff then $\left(E, \mathscr{T}^{\prime}\right)$ is Hausdorff if and only if $\dot{S}_{i}(x)=\theta$ for all $i \in I$ implies $x=\theta$. If $(F, \mathscr{T})$ is locally convex and is generated by a family of seminorms $P=\{p\}$ then $\mathscr{T}^{\prime}$ is generated by the family of seminorms $P^{\prime}=\left\{p^{\prime}\right\}$ where for each $p \in P$, $x \in E, p^{\prime}(x)=\sup _{i \in I} p\left(S_{i}(x)\right)$.

We sketch the proof of Theorem 4.4 for the case of a weak basis. Let $\left\{S_{n}\right\}_{n=1}^{\infty}$ denote the family of partial sum operators of the weak basis. Since for each $x \in E, S_{n}(x)$ converges weakly to $x$ it follows that $\left\{S_{n}(x): n \in \omega\right\}$ is bounded for each $x \in E$. By the lemma, there is a weakest vector topology $\mathscr{T}^{\prime}$ for $E$ such that the family $\left\{S_{n}\right\}$ is $\mathscr{T}^{\prime}-\mathscr{T}$ equicontinuous. By the Lemma $\mathscr{T}^{\prime}$ is metrizable and locally convex. Using the fact that $\left\{S_{n}\right\}_{n \in \omega}$ are the partial sum operators of a weak basis it can further be shown that $\mathscr{T} \subset \mathscr{T}^{\prime}$ and $\mathscr{T}^{\prime}$ is complete. Thus, $E$ has the two topologies $\mathscr{T}$ and $\mathscr{T}^{\prime}$ each making $E$ a Fréchet space and with $\mathscr{T} \subset \mathscr{T}^{\prime}$. By the open mapping theorem $\mathscr{T}=\mathscr{T}^{\prime}$. Thus the partial sum operators are continuous in the initial topology which implies the coefficient functionals are continuous in the initial and, hence, the weak topology. Applying Theorem 4.3 we conclude that the weak basis is a Schauder basis for $E$.

G. Bennett and J. B. Cooper [7] have shown that the weak basis and continuity theorems are valid for (LF) spaces.

Two other papers at about the same level of generality were published in the same year, 1969. One by K. Floret [32] stated that every basis in a sequentially retractive (LF) space is a Schauder basis. The other by M. De Wilde [24] stated that a weak basis in a bornological sequentially complete and strictly netted space is a Schauder basis. 
Dubinsky and Retherford [28] on the other hand give an example of a Mackey space with a weak Schauder basis which is not a basis. By a slight reformulation of their example, we note a better conclusion can be drawn. Namely, there exists a bornological space (hence, a Mackey space) with a weak Schauder basis which is not a basis in the initial topology of the space. The example is the following:

Let $(c)$ denote the Banach space of real convergent sequences with $\|x\|=\sup _{i \in \omega}\left|\xi_{i}\right|$ if $x=\left\{\xi_{i}\right\}$. Let $E$ denote the subspace of $(c)$ consisting of those elements of $(c)$ which are constant from some index on. Let $E$ have the norm topology which it inherits from $(c)$. Now $l^{1}$ is the conjugate space of both $E$ and $(c)$. It is easy to show that the unit vectors $\left\{l_{i}\right\}$ $\left(l_{i}=\left\{\delta_{i j}\right\}\right)$ are a weak Schauder basis for $E$. However, the vector $l=\left\{\xi_{i}\right\}$ where $\xi_{i}=1$ for all $i \in \omega$ is in $E$ but not in the strongly closed linear span of $\left\{l_{i}\right\}$ so $\left\{l_{i}\right\}$ is not a norm basis of $E$.

W. J. Stiles [114] has shown that for each $p, 0<p<1, l^{p}$ (a complete metric linear space) contains a weak Schauder basis which is not a basis.

On the positive side, it has been observed [77] that if $E$ is a locally convex space which is weakly sequentially complete, e.g. a reflexive space, then a weak unconditional basis for $E$ is an unconditional basis for $E$.

5. Several theorems of James. In 1950 [45], [46], R. C. James created and used the basis theory needed to settle an outstanding conjecture of that time. He disproved the conjecture that a Banach space $E$ is reflexive if and only if $E$ and $E^{\prime \prime}$ are isometric or $E^{\prime \prime}$ is separable. ${ }^{5}$

We will call a Schauder basis $\left\{x_{i}, f_{i}\right\}$ for, a Hausdorff topological vector space $E$ shrinking (Tumarkin [115] uses the perhaps more appropriate word "stretching") if $f_{i}$ is a basis for $E^{\prime}$ with its strong topology. This definition is easily seen to be equivalent to James's different definition for the Banach space case. Following Day [21] in terminology the Schauder basis $\left\{x_{i}, f_{i}\right\}$ is boundedly complete iff the series $\sum_{i=1}^{\infty} a_{i} x_{i}$ converges for each sequence of scalars $\left\{a_{i}\right\}$ for which the partial sums $\left\{\sum_{i=1}^{n} a_{i} x_{i}\right\}_{n=1}^{\infty}$ are bounded. James showed that a Banach space with a basis is reflexive if and only if the basis is boundedly complete and shrinking. He then constructed a Banach space, now called the James space $J$, with a basis which is shrinking but not boundedly complete. Furthermore, $J$ is isometric with $J^{\prime \prime}$. Moreover, the canonical mapping of $J$ into $J^{\prime \prime}$ has codimension 1 .

James's characterization of reflexivity has been successively generalized by Sanders [97], Retherford [95], Dubinsky and Retherford [29], and Cook [17], and Johnson [48].

\footnotetext{
${ }^{5}$ It is interesting to note that only recently [50] has it been proved that if $E$ is infinite dimensional and $E^{\prime \prime}$ is separable, then $E$ contains an infinite dimensional reflexive subspace.
} 
To state Cook's theorem we define a Schauder decomposition (equivalent to the notion of basis of subspaces defined earlier when the subspaces are closed) of $E$ as a sequence of continuous projections $\left\{P_{k}\right\}$ on $E$ together with their ranges $\left\{R\left(P_{k}\right)\right\}$ which satisfy the properties: $P_{k} \circ P_{j}=0$ when $k \neq j$ and for each $x \in E, x=\sum_{k=1}^{\infty} P_{k}(x)$. A Schauder decomposition of $E$ will be denoted by $\left\{R\left(P_{k}\right), P_{k}\right\}$. If each $R\left(P_{k}\right)$ is one dimensional, i.e., $R\left(P_{k}\right)$ is the linear span of a single nonzero element $x_{k} \in E$, then the decomposition is actually representable as a Schauder basis $\left\{x_{k}, f_{k}\right\}$ for $E$ with $f_{k}(x)$ defined by the equations $f_{k}(x) x_{k}=P_{k}(x), k=1,2, \ldots, x \in E$. Analogous to the basis case a Schauder decomposition is boundedly complete if for each bounded sequence $\left\{\sum_{k=1}^{n} x_{k}\right\}_{n=1}^{\infty}, x_{k} \in R\left(P_{k}\right)$, the series $\sum_{k=1}^{\infty} x_{k}$ converges. The Schauder decomposition $\left\{R\left(P_{k}\right), P_{k}\right\}$ is shrinking iff $\left\{R\left(P_{k}^{\prime}\right), P_{k}^{\prime}\right\}$ is a Schauder decomposition for $E^{\prime}$ with the strong topology. Cook's theorem is

THEOREM 5.1. Let $E$ be a locally convex space with a Schauder decomposition $\left\{R\left(P_{k}\right), P_{k}\right\} . E$ is semireflexive, i.e. the canonical mapping of $E$ into the space of strongly continuous linear functionals on $E^{\prime}$ is onto, if and only if $R\left(P_{k}\right)$ is semireflexive $(k=1,2, \ldots)$ and $\left\{R\left(P_{k}\right), P_{k}\right\}$ is boundedly complete and shrinking.

W. B. Johnson [48] has extended James's theorem to Markushevich bases ( $M$-bases). Johnson calls an $M$-basis $\left\{x_{i}, f_{i}\right\} M$-shrinking if the strongly closed linear span of $\left\{f_{i}\right\}$ is $E^{\prime}$. Also an $M$-basis $\left\{x_{i}, f_{i}\right\}$ is boundedly complete iff whenever $\left\{y_{d}\right\}$ is a bounded net in $E$. such that for each $i \in I$, $\lim _{d} f_{i}\left(y_{d}\right)$ exists, there is an $x \in E$ such that $f_{i}(x)=\lim _{d} f_{i}\left(y_{d}\right)$ for each $i \in I$. Johnson proves

THEOREM 5.2. Let $\left\{x_{i}, f_{i}\right\}$ be an $M$-basis for a locally convex space $E$. $E$ is semireflexive iff $\left\{x_{i}, f_{i}\right\}$ is $M$-shrinking and M-boundedly complete.

Cook's theorem for the Schauder basis case follows from Johnson's theorem.

James [45, Lemma 1] showed that an unconditional basis for a Banach space is boundedly complete if the space contains no subspace isomorphic to $c_{0}$. He also showed [45, Lemma 2] that a Banach space with an unconditional basis is shrinking provided it has no subspace isomorphic to $l^{1}$.

Day [21, pp. 74, 75] gives the following improvements of James's lemmas.

THEOREM 5.3. If $\left\{x_{i}\right\}$ is an unconditional basis for a Banach space $E$ the following are equivalent:

(a) The basis is boundedly complete.

(b) The space is weakly sequentially complete.

(c) There is no subspace of $E$ isomorphic to $c_{0}$. 
THEOREM 5.4. An unconditional basis for a Banach space $E$ is shrinking iff E has no subspace isomorphic to $l^{1}$.

Further improvements of James's theorems have been made by several people. L. J. Weill [116, Theorems 2.6, 2.7, 2.11] has shown

THEOREM 5.5. Let E be a barrelled space with an unconditional Schauder basis. Then the statements below are equivalent:

(1) The basis is boundedly complete.

(2) $E$ is weakly sequentially complete.

(3) $E$ is sequentially complete and contains no subspace isomorphic to $c_{0}$.

THEOREM 5.6. If $E$ is a sequentially complete barrelled space with an unconditional Schauder basis, the basis is shrinking if and only if $E$ has no subspace isomorphic to $l^{1}$.

The equivalence of (1) and (2) of Theorem 5.5 was proved by Dubinski and Retherford [29] who substituted for the hypothesis that $E$ is barrelled the hypothesis that the basis is a bounded multiplier basis, and $E^{\prime}$ is $\omega\left(E^{\prime}, E\right)$ sequentially complete. On the other hand, Tumarkin $[115$, Corollary to Theorem 4] has shown that if $E$ is sequentially complete (but not necessarily barrelled) then (1) and (3) of Theorem 5.5 are equivalent. Tumarkin [115, Theorem 5] has also shown that if an unconditional basis of a sequentially complete locally convex space $E$ is not shrinking, then $E$ contains a subspace $E_{1}$ which, when endowed with a certain topology related to the relative topology, is isomorphic to $l^{1}$.

Weill's results above yield the following improvement of a theorem of James [45, Theorem 2].

THEOREM 5.7. A sequentially complete barrelled space with an unconditional basis is reflexive iff it contains no subspaces isomorphic to $l^{1}$ or $c_{0}$.

Singer [107] showed that a Banach space in which every basic sequence is boundedly complete or every basic sequence is shrinking is reflexive. Zippin [120] then showed that a Banach space with a basis with the further property that every basis is boundedly complete or every basis is shrinking is reflexive.

Kalton [56] has given the following improvements of these theorems:

THEOREM 5.8. Let $E$ be a sequentially complete locally convex space possessing a Schauder basis.

(i) If every Schauder basic sequence in $E$ is boundedly complete, then $E$ is semireflexive.

(ii) If every Schauder basic sequence in $E$ is shrinking, then $E$ is semireflexive. 
A Schauder basis $\left\{x_{n}, f_{n}\right\}$ is semi-shrinking if there exists a neighborhood $V$ of zero such that $x_{n} \notin V$ for all $n$ and $\lim _{n \rightarrow \infty} x_{n}=0$ weakly. A Schauder basis $\left\{x_{n}, f_{n}\right\}$ is semi-boundedly-complete iff $\left\{x_{n}\right\}$ is bounded and whenever $\left\{\sum_{i=1}^{n} \lambda_{i} x_{i}\right\}_{n=1}^{\infty}$ is bounded then $\lim _{n \rightarrow \infty} \lambda_{n}=0$.

THEOREM 5.9. Let $E$ be a complete barrelled space possessing a normalized Schauder basis $\left\{x_{n}, f_{n}\right\}$ (i.e. $\left\{x_{n}\right\}$ is bounded and for some neighborhood $V$ of $0, x_{n} \notin V$ for all $n$ ). The following are equivalent:

(i) Every normalized Schauder basis is shrinking.

(ii) Every normalized Schauder basis is semishrinking.

(iii) Every normalized Schauder basis is boundedly-complete.

(iv) Every normalized Schauder basis is semi-boundedly-complete.

(v) $E$ is reflexive.

6. Existence of bases. I. Singer [111] pointed out that the $w^{*}$-dual of the Banach space $m$ of all bounded sequences is a separable locally convex space which does not admit a $w^{*}$-Schauder basis. N. J. Kalton [57] has recently given a more incisive example.

A topological vector space $E$ will be called $\omega$-separable iff it possesses a subspace $G$ of countable dimension such that every member of $E$ is the limit of a sequence in $G$. If $E$ has a basis, $E$ is $\omega$-separable and if $E$ is $\omega$-separable, then $E$ is separable. Let $\times$ denote the cardinal of the continuum, and let $K$ be the field of real or complex numbers. Kalton shows

TheORem 6.1. $K^{\mathrm{K}}$ is a complete bornological, nuclear, Ptak space; it is separable but not $\omega$-separable, and so does not possess a basis.

Note too that since $K^{\mathrm{x}}$ is a nuclear space it is a Montel space and hence a reflexive space and, thus, is barrelled as well.

On the other hand, there are a number of separable Banach spaces for which bases have not been shown to exist [112, p. 17]. When Banach posed the basis problem, he also asked [6, p. 238] if the space $C^{(1)}\left(I^{n}\right)$ has a basis. Not until 1969 was an affirmative answer given to the question by S. Schonefeld [102] and Z. Ciesielski [16], working independently. In 1970, P. Billard [14] announced there is a basis for the Hardy space $H^{1}$ as well as a Schauder basis of finite dimensional subspaces of the disc algebra $A$.

The strongest known general result on the existence of a basis in a class of spaces which do not have an explicit presentation as function spaces is due to Johnson, Rosenthal, and Zippin [51].

THEOREM 6.2. Every separable $\mathscr{L}^{p}$ space, $1 \leqq p<\infty$, has a basis. In particular every complemented subspace of $C[0,1]$ and $L^{p}[0,1], 1 \leqq p<\infty$, has a basis. 
If every separable Banach space had a basis, it would, of course, be true that every separable Banach space would have a Schauder decomposition into finite dimensional subspaces. However, it is not yet known whether a separable Banach space admits a Schauder decomposition of any kind. Inasmuch as nonseparable Banach spaces can have Schauder decompositions the question "Does every infinite dimensional Banach space admit a Schauder decomposition?" is of interest. Dean [23] gave a negative answer to the question.

THEOREM 6.3. The Banach space $(m)$ does not admit a Schauder decomposition.

In finite dimensional spaces, all bases are unconditional. It is not difficult to exhibit conditional bases for some of the classical infinite dimensional Banach spaces. For example [112, p. 423] in the space $c_{0}$ the vectors

$$
x_{n}=\{\underbrace{1, \ldots, 1}_{n}, 0,0, \ldots\}, \quad n=1,2, \ldots,
$$

are a conditional basis whereas the unit vector basis for $c_{0}$ is unconditional. It was natural to ask "Does every separable Banach space admit an unconditional basis?" In 1948 S. Karlin [58] showed that $C[0,1]$ does not admit an unconditional basis. This result was improved in 1958 by Bessaga and Pelczynski [10] who showed

THEOREM 6.4. A separable Banach space having the space $J$ of James as a subspace, e.g. C $[0,1]$, does not have an unconditional basis.

It was also shown independently by A. Pelczynski [88] and I. Singer $[105],[106]$ that

THEOREM 6.5. Any weakly sequentially complete Banach space not isomorphic to a conjugate space (e.g. $L[0,1])$ does not have an unconditional basis.

On the positive side, J. E. Shirey and R. E. Zink [104] have recently proved a generalization of the following theorem of Gaposhkin [36]:

THEOREM 6.6. The Haar functions form an unconditional basis for the reflexive Orlicz spaces.

On the other hand, the following was shown recently by A. Pelczynski and S. Kwapien [92] and also by Lindenstrauss and Pelczynski [67].

THEOREM 6.7. There exists a separable reflexive Banach space which does not admit an unconditional basis. 
Since it has been established that there exist spaces in which all bases are conditional, it is natural to ask "Do there exist infinite dimensional Banach spaces in which all bases are unconditional?" In a beautiful paper, A. Pelczynski and I. Singer [93] show

THEOREM 6.8. In every infinite dimensional Banach space with a basis there exist nonequivalent bases. (Bases $\left\{x_{i}\right\}$ and $\left\{y_{i}\right\}$ are equivalent iff $\left\{\left\{a_{i}\right\}: \sum_{i=1}^{\infty} a_{i} x_{i}\right.$ converges $\}=\left\{\left\{a_{i}\right\}: \sum_{i=1}^{\infty} a_{i} y_{i}\right.$ converges $\left.\}.\right)$

Theorem 6.8 is a main lemma for the following outstanding result:

THEOREM 6.9. Every separable infinite dimensional Banach space with a basis has a conditional basis.

In order to prove the above two theorems, the notions of symmetric and subsymmetric bases enter in a natural and fundamental way.

A basis $\left\{x_{n}\right\}$ for a space $E$ is symmetric iff for each permutation $\tau$ of $\omega$ the sequence $\left\{x_{\tau(n)}\right\}$ is a basis for $E$ equivalent to $\left\{x_{n}\right\}$.

A basis $\left\{x_{n}\right\}$ for a space $E$ is subsymmetric iff it is an unconditional basis and is equivalent to each of its subsequences $\left\{x_{n_{i}}\right\}$.

It is known that a symmetric basis for a Banach space is subsymmetric and Garling [37] has given an example of a Banach space with a subsymmetric basis which is not symmetric.

A brief sketch of the proof of the Pelczynski-Singer theorem on the existence of conditional bases may be illuminating. Suppose that $E$ is an infinite dimensional Banach space such that all normalized bases $\left\{x_{i}\right\}$ for $E$ are equivalent. Let $\left\{x_{n}\right\}$ be a normalized basis for $E$, i.e., $\left\{x_{n}\right\}$ is a basis such that $\left\|x_{n}\right\|=1, n=1,2, \ldots$. If $\left\{\varepsilon_{n}\right\}$ is an arbitrary sequence with $\varepsilon_{n}= \pm 1, n=1,2, \ldots$, it follows that $\left\{\varepsilon_{n} x_{n}\right\}$ is a normalized basis of $E$. If all normalized bases of $E$ are equivalent, it follows that $\sum_{i=1}^{\infty} a_{i} x_{i}$ converges if and only if $\sum_{i=1}^{\infty} \varepsilon_{i} a_{i} x_{i}$ converges for arbitrary $\left\{\varepsilon_{n}\right\}, \varepsilon_{n}= \pm 1$. Thus $\left\{x_{n}\right\}$ is an unconditional normalized basis. Hence, for each permutation $\tau$ of $\omega,\left\{x_{\tau(n)}\right\}$ is a normalized basis which, by assumption, must be equivalent to $\left\{x_{n}\right\}$. Thus $\left\{x_{n}\right\}$ is a symmetric basis. Using notions of generalized Haar system, generalized Rademacher system, and generalized Khinchin inequality plus some facts about block basic sequences, it is shown that if all normalized bases are equivalent, then $E$ is isomorphic to $l^{2}$. But $\left\{x_{n}\right\}$ is an unconditional basis for $E$ and $l^{2}$ has been shown by Babenko [4] to have a conditional normalized basis. Since $E$ cannot have equivalent bases one of which is unconditional, the other conditional, we must conclude that $E$ admits two nonequivalent normalized bases. This fact together with more use of symmetric and subsymmetric bases leads to the "construction" of a conditional basis for $E$.

I. Singer [112, p. 587] asked "In a Banach space $E$ with a symmetric basis are all symmetric bases equivalent?" Lindenstrauss and Tzafriri 
[69] have recently constructed a reflexive Orlicz space having at least two nonequivalent symmetric bases.

A special case of the basis problem which should be more tractable than the general question but nevertheless remains open is: Does every separable uniformly convex space have a basis?

Although not much work has been done on it, the basis problem for finite dimensional spaces is important. Given a basis $x_{1}, x_{2}, \ldots, x_{n}$ in a Banach space of dimension $n$, define the basis constant of $x_{1}, \ldots, x_{n}$ to be the smallest $K$ such that for all scalars $a_{1}, \ldots, a_{n}$ and $1 \leqq j<n$, $\left\|\sum_{i=1}^{j} a_{i} x_{i}\right\| \leqq K\left\|\sum_{i=1}^{n} a_{i} x_{i}\right\|$. The basis problem for finite dimensional spaces is: Does there exist a $K<\infty$ so that every finite dimensional Banach space has a basis with constant no greater than $K$ ?

Of course, the above question is open for infinite dimensional Banach spaces as well. In this connection P. Enflo [31] has shown: There exists an infinite dimensional Banach space $E$ and a number $\lambda>1$ such that if $E$ has a basis, then any basis for $E$ has basis constant greater than $\lambda$.

Similarly one can define the unconditional basis constant of a basis for a finite dimensional space. Does there exist a $K<\infty$ such that every finite dimensional space has a basis with an unconditional basis constant no greater than $K$ ? The answer is very likely no. Garling and Gordon [38] have shown that if the word "unconditional" is replaced by "symmetric" the answer to the question is indeed no.

7. Block basic sequences. Let $\left\{x_{n}\right\}$ be a basis for a Banach space $E$, let $\left\{m_{n}\right\}$ be an increasing sequence of positive integers, $m_{0}=0$, and let $y_{n}=\sum_{i=m_{n-1}+1}^{m_{n}} a_{i} x_{i}, y_{n} \neq 0(n=1,2, \ldots)$. Then $\left\{y_{n}\right\}$ is a basic sequence $\left[112\right.$, p. 66]. A sequence $\left\{y_{n}\right\} \subset E$ constructed in the above manner from a basis $\left\{x_{n}\right\}$ is said to be a block basic sequence with respect to the basis $\left\{x_{n}\right\}$. Block basic sequences were used by James [45] and by Bessaga and Pelczynski [9] who gave them their name.

The notion of a block basic sequence with respect to a basis is central in much recent work in basis theory. The following are some results based on this notion.

THEOREM 7.1 (ZIPPIN [119]). The unit vector bases of $c_{0}$ and $l^{p}, 1 \leqq p$ $<\infty$, are the only perfectly homogeneous bases for Banach spaces, i.e., bases which are equivalent to each of their normalized block bases.

THEOREM 7.2 (LiNDENSTRAuSs-ZIPPIN [70]). The only Banach spaces which have, up to equivalence, unique, unconditional, normalized bases are $c_{0}, l^{1}$, and $l^{2}$.

The fact that $l^{2}$ has a unique normalized unconditional basis is classical going back to Köthe-Toeplitz [62] in 1934 and E. Lorch [71] in 1939. For $c_{0}$ and $l^{1}$ this was proved by Lindenstrauss and Pelczynski [66] and 
is an easy consequence of Grothendieck's inequality. The converse is based on block basis techniques and leans heavily on Theorem 7.1 above.

Corollary 7.3 (PelCZynski-Singer [93]). If $E$ is an infinite dimensional Banach space with a basis, then $E$ admits nonequivalent bases.

Proof. Suppose all normalized bases for $E$ are equivalent. Let $\left\{x_{n}\right\}$ be a normalized basis for $E$. If $\left\{\varepsilon_{n}\right\}$ is an arbitrary sequence with $\varepsilon_{n}= \pm 1$, $n=1,2, \ldots$, it follows that $\left\{\varepsilon_{n} x_{n}\right\}$ is a normalized basis for $E$. Since all normalized bases for $E$ are equivalent, it follows that $\sum_{i=1}^{\infty} a_{i} x_{i}$ converges iff $\sum_{i=1}^{\infty} \varepsilon_{i} a_{i} x_{i}$ converges for arbitrary $\left\{\varepsilon_{n}\right\}, \varepsilon_{n}= \pm 1, n=1,2, \ldots$. Thus $\left\{x_{n}\right\}$ is an unconditional normalized basis for $E$. From our assumption, we have that $E$ is a space with a normalized unconditional basis and all normalized unconditional bases for $E$ are equivalent. Thus $E$ is isomorphic to $c_{0}, l^{1}$, or $l^{2}$. Now the unit vector basis for each of these spaces is unconditional whereas it is classical that each of these spaces has a normalized conditional bases. Since these bases cannot be equivalent, we have arrived at a contradiction.

THEOREM 7.4 (ZIPPIN [120]). Every block basic sequence of a basis for a Banach space $E$ can be extended to a basis for $E$.

The above result enabled Zippin to complete the by now classical results of James by showing that if a Banach space $E$ has a normalized basis and every basis for $E$ is shrinking (respectively boundedly complete) then $E$ is reflexive.

THeOREM 7.5 (PelCZyNSKI-Singer [93]). Let $\left\{x_{n}\right\}$ be a normalized nonsymmetric unconditional basis for a Banach space $E$. Then there exists a block perturbation of a suitable permutation of $\left\{x_{n}\right\}$ which is a conditional basis for $E$.

We now give an alternate proof $[112$, p. 620] of the Pelczynski-Singer theorem of the preceding section.

THEOREM 7.6. Every infinite dimensional Banach space $E$ with a basis has a conditional basis.

Proof. Suppose all normalized bases for $E$ are unconditional. If there exists a normalized nonsymmetric unconditional basis for $E$ then, by Theorem 7.5., $E$ has a normalized conditional basis so we have a contradiction in this case. Suppose, on the other hand, that all normalized unconditional bases for $E$ are symmetric. Let $\left\{x_{n}\right\}$ be a normalized, unconditional and hence symmetric basis for $E$. It may be shown [112, p. 619] that $\left\{x_{n}\right\}$ is equivalent to all of its normalized block basic sequences, i.e., $\left\{x_{n}\right\}$ is perfectly homogeneous and by Theorem 7.1, $E$ is 
isomorphic to $c_{0}$ or $l^{p}, 1 \leqq p<\infty$. Since these spaces have normalized conditional bases, we again have reached a contradiction.

TheOREM 7.7 (Lindenstrauss-TZAFriRI [68]). If $\left\{x_{i}\right\}$ is a symmetric basis for a Banach space and every block basic sequence with respect to $\left\{x_{i}\right\}$ spans a complemented subspace of $E$ then $\left\{x_{i}\right\}$ is perfectly homogeneous.

It follows from the above that the unconditional analog of Theorem 7.4 fails badly unless $E$ is $c_{0}$ or $l^{p}, 1 \leqq p<\infty$.

Usually a block basis with respect to a basis $\left\{x_{n}\right\}$ inherits the nice properties of $\left\{x_{n}\right\}$. This observation led Lindenstrauss and Pelczynski [67] to define the notion of a reproducing basis (see [67] for definition) which, in a sense, is better (perhaps more precisely, not worse) than any other basis of the same space. Not every space with a basis has a reproducing basis and, on the other hand, a reproducing basis need not be unique (up to equivalence). However, the notion of reproducing basis is useful. For example, it enabled Lindenstrauss and Pelczynski to give a simple proof of the existence of a reflexive space which does not have an unconditional basis. It is useful in handling "distorted norms" and will surely find many other applications in the future. Among the examples of reproducing bases are symmetric bases, the Haar basis in $L^{p}[0,1]$, and every basis for $C[0,1]$.

Lindenstrauss and Tzafriri [68] use results of basis theory in a critical way to present an affirmative solution to the question "If every closed subspace of a Banach space is complemented is the space a Hilbert space?" They also give the simplest proof of the Pelczynski-Singer Theorem 7.6. Their proof depends upon Theorem 7.2 and block bases.

8. Partial orderings determined by biorthogonal systems. In 1954 at the International Congress of Mathematicians at Amsterdam, R. E. Fullerton initiated the study of partial orderings determined by biorthogonal systems with his paper Geometric properties of a basis in a Banach space [34]. Some definitions are needed in order to discuss this development. Throughout this section $E$ will denote a real Hausdorff topological vector space and $\left\{x_{i}, f_{i}\right\}$ a biorthogonal system $\left\{x_{i}\right\} \subset E,\left\{f_{i}\right\} \subset E^{\prime}$. Three closely related wedges in $E$ associated with $\left\{x_{i}, f_{i}\right\}$ will be considered. They are

$$
\begin{aligned}
& K_{c}=\left\{x \in E: x=\sum_{i=1}^{\infty} f_{i}(x) x_{i} \text { and } f_{i}(x) \geqq 0, i \in \omega\right\}, \\
& K_{0}=\{x \in E: x \text { is in the closure of the set of elements of the form } \\
& K=\left\{x \in E: f_{i}(x) \geqq 0, i \in \omega\right\} .
\end{aligned}
$$


It is clear that $K_{0}$ and $K$ are closed and $K_{c} \subset K_{0} \subset K$ and when $\left\{x_{i}, f_{i}\right\}$ is a basis the three wedges are equal. Recall that for any wedge $P$ in $E$ a partial order $\leqq_{p}$ may be defined for $E$ by $x \leqq_{p} y$ if $y-x \in P$. This partial ordering is antisymmetric when the wedge $P$ is a cone, i.e., $P \cap(-P)$ $=\{\theta\}$. The wedge $K$ defined above is a cone iff $\left\{f_{i}\right\}$ is total. A nonempty convex set $B$ is called a base of a wedge $P$ iff each nonzero element $x$ of $P$ has a unique representation of the form $x=\lambda b, \lambda>0, b \in B$. Fullerton ([34], [35]) observed that the cone $K$ of a basis $\left\{x_{i}, f_{i}\right\}$ has no interior points and does not admit a compact or even a weakly compact base.

If the cone of a basis for an infinite dimensional space cannot have a weakly compact base, can it have a bounded base? It was recently shown by Singer, McArthur, and Levin [80] that every basis cone in an infinite dimensional Banach space has an unbounded base. On the other hand, if $\left\{x_{n}, f_{n}\right\}$ is a Schauder basis for a Banach space $E$ with $\left\|x_{n}\right\|=1, n \in \omega$, and $K_{\left\{x_{n}\right\}}$ is the cone of the basis, then it was shown [80] that $K_{\left\{x_{n}\right\}}$ has a bounded base iff the mapping $T\left(\sum_{i=1}^{\infty} a_{i} x_{i}\right)=\sum_{i=1}^{\infty} a_{i} l_{i}$ is well defined from $K_{\left\{x_{n}\right\}}$ onto $K_{\left\{l_{n}\right\}}$ where $\left\{l_{n}\right\}$ is the unit vector basis of $l^{1}$ and $K_{\left\{l_{n}\right\}}$ is the cone of $\left\{l_{n}\right\}$. Foias, and Singer [112, p. 323] show that when $T$ is well defined it is necessarily bicontinuous. From the foregoing results, the following theorem $[\mathbf{8 0}]$ is clear.

THEOREM 8.1. If $\left\{x_{n}, f_{n}\right\}$ is a basis for a Banach space $E$ with $\left\|x_{n}\right\|=1$, $n \in \omega$, then $\left\{x_{n}\right\}$ is equivalent to the unit vector basis of $l^{1}$ iff $K_{\left\{x_{n}\right\}}$ has a bounded base and generates $E$ (i.e., $E=K-K$ ).

S. Saxon [98] has made a study of bases of cones of biorthogonal systems. He has shown, for example, that if $K$ is the cone of a Schauder basis $\left\{x_{i}, f_{i}\right\}$ of a locally convex Hausdorff space $E$ and $K-K$ is barrelled, then $K$ has a bounded base iff $E$ is, by identification, a barrelled subspace of $l^{1}$ which contains $\left\{l_{i}\right\}$ as a "normalization" of $\left\{x_{i}\right\}$.

Although Fullerton's announcement in 1954 at the International Congress is the earliest reference we have found on orderings by bases, the first published paper is that of $\mathrm{H}$. Schaefer in 1958 [99]. In this paper Schaefer develops the general theory of partially ordered locally convex spaces and he then makes application of the general theory to the special case of locally convex spaces which are ordered by biorthogonal systems.

If $P$ is a wedge in a vector space $E$, then a set $A$ is order convex (with respect to $P$ ) iff $A$ contains each order interval of $P$ whose end points are in $A$. A wedge $P$ in a topological vector space $E$ is normal iff $E$ has a local base of $P$-order convex neighborhoods of zero. A normal wedge in a Hausdorff space is necessarily a cone. A wedge $P$ in $E$ is a $b$-wedge iff each bounded set in $E$ is contained in the closure of a set of the form $(B \cap P)-(B \cap P)$ where $B$ is a bounded set. A wedge $P$ is a strict $b$-wedge iff each bounded set is contained in a set of the form $(B \cap P)-(B \cap P)$ 
where $B$ is bounded. Following Singer $[112$, p. 24$]$ we say that a biorthogonal system $\left\{x_{n}, f_{n}\right\}$ for $E$ is $E$-complete iff the set of finite linear combinations $\sum_{i=1}^{n} a_{i} x_{i}\left(n \in \omega, a_{1}, a_{2}, \ldots, a_{n}\right.$ arbitrary scalars $)$ is dense in $E$.

The following theorem gives a number of order theoretic characterizations for a biorthogonal system to be an unconditional basis. It includes results obtained by Schaefer [99, p. 139], Singer [107, p. 251], Gurevich [43], Ceitlin [15, Theorem 7] and Levin and McArthur [64].

THEOREM 8.2. Let $\left\{x_{n}, f_{n}\right\}$ be an E-complete biorthogonal system, for a barrelled space $E$ and let $P$ denote any of the wedges $K_{c}, K_{0}$, or $K$. The following are equivalent:

(i) $\left\{x_{n}, f_{n}\right\}$ is an unconditional basis for $E$.

(ii) $P$ is a normal b-cone.

(iii) $P$ is normal and $P^{\prime}$ is strongly normal.

(iv) $P^{\prime}$ generates $E^{\prime}$ and $[0, f]$ is bounded for each $f \in P^{\prime}$.

Conditions (i) through (iv) are implied by each of the following conditions and all eight conditions are equivalent when $E$ is sequentially complete.

(v) $P$ generates $E$ and $[0, x]$ is bounded for each $x \in P$.

(vi) $P$ generates $E$ and $P^{\prime}$ generates $E^{\prime}$.

(vii) $P$ is normal and generating.

(viii) $P$ is a normal strict $b$-cone.

The condition that $\left\{x_{n}, f_{n}\right\}$ be $E$-complete cannot in general be dropped from the above theorem [112, p. 478].

In his paper of 1962 Fullerton [35] proved that for a sequentially complete locally convex space $E$ ordered by the cone of an unconditional basis each order interval is homeomorphic to a countable Hilbert cube. Thus the order intervals are compact and metrizable. Implicit in his paper is the result

Proposition 8.3. A biorthogonal system $\left\{x_{n}, f_{n}\right\}$ with $\left\{f_{n}\right\}$ total in a sequentially complete locally convex space $E$ is an unconditional basis for $E$ iff its cone $K$ is generating and the order intervals determined by $K$ are compact.

\section{Nguen Van Khue [59] showed}

Proposition 8.4. A biorthogonal system $\left\{x_{n}, f_{n}\right\}$ with $\left\{f_{n}\right\}$ total in a Banach space $E$ is an unconditional basis for $E$ iff its cone $K$ is generating and each of the order intervals $[0, x], x \in K$, is weakly compact in a certain auxiliary topology.

The next theorem, due to the author [78], includes Proposition 8.3. A wedge $P$ in a topological vector space $E$ is regular iff each monotone nondecreasing net in $P$ which is majorized by an element of $P$ converges. If the above condition is satisfied by sequences, $P$ is sequentially regular. 
THEOREM 8.5. Let $E$ be a locally convex Hausdorff space and $\left\{x_{n}, f_{n}\right\} a$ biorthogonal system in $E$ with $\left\{f_{n}\right\}$ total and let $E$ be partially ordered by the cone $K$. Then the following are equivalent:

(i) For each $x \in K$ and each bounded sequence $\left\{b_{i}\right\}$ of nonnegative real numbers the series $\sum_{i=1}^{\infty} b_{i} f_{i}(x) x_{i}$ converges.

(ii) $[0, x]$ is compact for each $x \in K$.

(iii) $[0, x]$ is weakly compact for each $x \in K$.

(iv) $K$ is weakly regular.

(v) $K$ is sequentially weakly regular.

(vi) $K$ is regular.

(vii) $K$ is sequentially regular.

(viii) $[0, x]$ is $\sigma\left(E, E^{\prime}\right)$-sequentially complete and bounded for each $x \in K$. Moreover, if $E$ is reflexive each of the above is equivalent to

(ix) $[0, x]$ is bounded for each $x \in K$.

The next two corollaries were proved by Gurevich [43] for Banach spaces.

COROLlary 8.6. A biorthogonal system $\left\{x_{n}, f_{n}\right\}$ with $\left\{f_{n}\right\}$ total in a sequentially complete locally convex space $E$ is an unconditional basis if and only if its cone $K$ is regular and generating.

COROLlary 8.7. A biorthogonal system $\left\{x_{n}, f_{n}\right\}$ in a reflexive space $E$ is an unconditional basis for $E$ iff its wedge $K$ is normal and generating.

Proof. Suppose that $\left\{x_{n}, f_{n}\right\}$ is an unconditional basis for $E$. Since $E$ is reflexive, $E$ is barrelled so the cone $K$ is normal [78, Lemma 7]. Also since $E$ is reflexive, $E$ is sequentially complete so given $x \in E$ let $x=y-z$ where $y$ is the sum of the subseries of $\sum_{i=1}^{\infty} f_{i}(x) x_{i}$ obtained by using the terms with $f_{i}(x) \geqq 0$ and $-z$ is the sum of the remaining terms. Conversely, if the wedge $K$ is normal, then $K$ must be a cone and hence $\left\{f_{i}\right\}$ is total. Also $K$ is closed so $K$ being closed and normal its order intervals are closed and bounded and since $E$ is reflexive, the order intervals are $\sigma\left(E, E^{\prime}\right)$-compact. From the equivalence of (i) and (iii) of Theorem 8.8 and the assumption that $K$ generates $E$ it follows that $\left\{x_{n}, f_{n}\right\}$ is an unconditional basis for $E$.

In the next theorem it is assumed that the biothogonal system converges on its cone $K$ (i.e., $K_{c}=K$ ) but not necessarily on the whole space. Although the theorem was originally stated and proved for Banach spaces [80] it is true for Fréchet spaces by virtually the same proof. The assumptions of the next theorem are satisfied by conditional as well as unconditional bases. For example, let $\left\{x_{n}, f_{n}\right\}$ denote Schauder's basis for $C[0,1]$. This basis is conditional since all bases for $C[0,1]$ are conditional. Also $K_{c}=K$ since it is a basis. Furthermore, $K$ is normal being a subset 
of the natural cone of positive elements of $C[0,1]$ which is well known to be normal.

THEOREM 8.8. Let $E$ be a Fréchet space and let $\left\{x_{n}, f_{n}\right\}$ be a biorthogonal system in $E$ such that $\left\{f_{n}\right\}$ is total and $K_{c}=K$. Then the following are equivalent:

(i) For every $x \in K, \sum_{i=1}^{\infty} f_{i}(x) x_{i}$ converges unconditionally.

(ii) For every $x \in K, f \in E^{\prime}, \sum_{i=1}^{\infty}\left|f_{i}(x) f\left(x_{i}\right)\right|<+\infty$.

(iii) $K$ is normal.

(iv) $[0, x]$ is bounded for each $x \in K$.

(v) For every $x \in K,[0, x]$ is linearly homeomorphic to a countable Hilbert cube. Moreover, if (i) holds $K$ is minihedral.

The above results show that the properties of regularity and normality of cones are related. In fact, in a Fréchet space every regular cone is normal. Moreover, it is known that every closed normal cone in a weakly sequentially complete locally convex space is regular. Other results in this direction are the following two theorems [79]:

THEOREM 8.9. If $E$ is a Fréchet space, the following are equivalent:

(i) Each closed normal cone in $E$ is regular.

(ii) E has no subspace isomorphic to $\left(c_{0}\right)$.

(iii) Each closed subspace of $E$ with an unconditional basis is weakly sequentially complete.

(iv) Each unconditionally basic sequence in $E$ is boundedly complete.

THEOREM 8.10. Let $E$ be a Banach space whose dual space $E^{\prime}$ with the strong topology is separable. Then with respect to the strong topology each closed normal cone in $E^{\prime}$ is regular.

COROLlARY 8.11. Let $E$ be the conjugate of a Banach space and assume that $E$ is strongly separable. If $\left\{x_{n}, f_{n}\right\}$ is a biorthogonal system in $E$ and $K$ is normal, then the series $\sum_{i=1}^{\infty} f_{i}(x) x_{i}$ is unconditionally convergent to $x$ for each $x \in K$.

Proof. By Theorem $8.10 K$ is regular and since $0 \leqq \sum_{i=1}^{n} f_{i}(x) x_{i}$ $\leqq \sum_{i=1}^{n+1} f_{i}(x) x_{i} \leqq x$, for $x \in K$ and $n=1,2, \ldots$, it follows that $\sum_{i=1}^{\infty} f_{i}(x) x_{i}$ converges. When $K$ is normal $\left\{f_{k}\right\}$ is total so the series must converge to $x$. The convergence is unconditional since applying the same argument as above to each subseries we see each subseries of $\sum_{i=1}^{\infty} f_{i}(x) x_{i}$ also converges.

It is easy to verify that if $\left\{x_{n}, f_{n}\right\}$ is an unconditional Schauder basis for a real sequentially complete locally convex space $E$, then $E$ with the partial ordering furnished by $K$ is a vector lattice. Furthermore, for each $x, y \in E$, 


$$
\max (x, y)=\sum_{i=1}^{\infty} \max \left(f_{i}(x), f_{i}(y)\right) x_{i}
$$

and

$$
\min (x, y)=\sum_{i=1}^{\infty} \min \left(f_{i}(x), f_{i}(y)\right) x_{i}
$$

and

$$
|x|=\sum_{i=1}^{\infty}\left|f_{i}(x)\right| x_{i} .
$$

I. Singer [112, pp. 559, 560] showed that if $E$ is a Banach space with an unconditional basis $\left\{x_{n}, f_{n}\right\}$ then $E$ ordered by $K$ may be renormed with an equivalent norm $\|x\|(x \in E)$ having the property that $|x| \leqq|y|$ implies $\|x\| \leqq\|y\|$ for $x, y \in E$, i.e., $E$ is a Banach lattice. Ceitlin [15] has shown that if $E$ is sequentially complete and bornological (hence barrelled as well) and $E$ is ordered by the cone $K$ of an unconditional Schauder basis, then $E$ is a locally convex lattice with the ordering determined by $K$, i.e. the lattice operations are continuous. J. Hofler [44] has taken this a step further with the following theorem.

THEOREM 8.12. Let E be a sequentially complete barrelled space ordered by the cone $K$ of an unconditional Schauder basis $\left\{x_{n}, f_{n}\right\}$. Then

(i) $E$ is a locally convex lattice;

(ii) the only complete barrelled space $E$ ordered by the cone of an unconditional Schauder basis for which the lattice operations are $\sigma\left(E, E^{\prime}\right)$ continuous is the space(s) of all real sequences with the product topology.

COROLlaRY 8.13. An arbitrary reflexive space ordered by the cone of an unconditional Schauder basis is a vector lattice.

Proof. A reflexive space is sequentially complete and barrelled.

S. Smith [113] has shown that if two biorthogonal systems have the same cone, they differ at most by rearrangement and positive scalar multiplication. Moreover, if $E$ is a Hausdorff topological vector space ordered by the cone $K$ of an unconditional Schauder basis $\left\{x_{n}, f_{n}\right\}_{n \in \omega}$ and if $\left\{y_{\alpha}, h_{\alpha}\right\}_{\alpha \in A}$ is a biorthogonal system having the same cone $K$ then $\omega=A$ and $\left\{y_{n}, h_{n}\right\}$ is an unconditional basis for $E$.

Marti and Sherbert [74] have recently proved the following weak basis theorem which involves an order topology. Let $E$ be a Fréchet space ordered by a generating cone. Let $o\left(E, E^{\prime}\right)$ denote the topology of uniform convergence on order bounded subsets of $E^{\prime}$. Then each weak $o\left(E, E^{\prime}\right)$ basis for $E$ is an $o\left(E, E^{\prime}\right)$ Schauder basis for $E$. 
9. Bases in nuclear Fréchet spaces. In the class of all infinite dimensional Banach spaces the Hilbert spaces seem to be the natural generalization of the finite dimensional normed linear spaces. However, in the class of all infinite dimensional Fréchet spaces, the nuclear spaces of A. Grothendieck [40] are the natural generalizations and nearest relatives of the finite dimensional spaces.

Grothendieck's original definition of nuclear spaces involves tensor products. A more elementary equivalent definition is the following. A sequence $\left\{x_{n}\right\}$ in a Fréchet space is rapidly decreasing iff $\left\{n^{k} x_{n}\right\}_{n=1}^{\infty}$ is bounded for each positive integer $k$. A set $N$ in a Fréchet space $E$ is a nuclear set iff $N$ is contained in the closed, convex, circled hull of some rapidly decreasing sequence. Finally, a Fréchet space is nuclear iff every bounded subset of $E$ is a nuclear set. Grothendieck [40] has shown that a Fréchet space $E$ is nuclear iff every unconditionally convergent series in $E$ is absolutely convergent.

The above characterization together with the fact that only in finite dimensional Banach spaces is unconditional convergence of series equivalent to absolute convergence implies that the only nuclear Banach spaces are the finite dimensional ones. Nuclear Fréchet spaces are necessarily separable. It is an open question as to whether or not every nuclear Fréchet space has a basis, i.e., the basis problem for nuclear Fréchet spaces is unsolved.

A major result in the theory of bases in nuclear spaces is due to A. S. Dynin and B. S. Mityagin [30]:

THEOREM 9.1. Each basis $\left\{x_{n}, f_{n}\right\}$ of a nuclear Fréchet space $E$ is absolute, i.e., if $x \in E$ and $p(x)$ is a continuous seminorm on $E$, then

$$
\sum_{n=1}^{\infty} p\left(f_{n}(x) x_{n}\right)<+\infty
$$

The above theorem allows one to represent each nuclear Fréchet space with a basis as a Köthe sequence space, which we now define. If $\Gamma$ is a set of positive sequences $a=\left\{a_{n}\right\}$ (i.e., $a_{n} \geqq 0, n=1,2, \ldots$ ) satisfying

(i) for each $n \in \omega$ there is an $a \in \Gamma$ such that $a_{n} \neq 0$, and

(ii) for $a^{1}, \ldots, a^{n} \in \Gamma$ there is an $a \in \Gamma$ and $M>0$ such that $a_{n}^{i} \leqq M a_{n}$ for all $i \in \omega$ and $n \in \omega$,

then let $\lambda(\Gamma)$ be the set of sequences of scalars $x=\left\{x_{n}\right\}$ such that $p_{a}(x)$ $\equiv \sum_{n=1}^{\infty} a_{n}\left|x_{n}\right|<+\infty$ for all $a \in \Gamma$. The vector space $\lambda(\Gamma)$ with the topology generated by the seminorms $\left\{p_{a}: a \in \Gamma\right\}$ is called a Köthe sequence space.

It is a consequence of the Dynin-Mityagin theorem above that a nuclear Fréchet space $E$ with a basis $\left\{x_{n}\right\}$ is isomorphic to the Köthe sequence space $\lambda(\Gamma), \Gamma=\left\{\left|x_{n}\right|_{m}\right\}$, where $\left\{|\cdot|_{m}\right\}_{m=1}^{\infty}$ is an increasing family of seminorms generating the topology of $E$. 
A converse of the Dynin-Mityagin theorem is valid. W. Wojtynski [117] has shown

THEOREM 9.2. If $E$ is a Fréchet space with a basis and all bases for $E$ are absolute, then $E$ is nuclear.

It has been conjectured that every Fréchet space in which all bases are unconditional is nuclear. In support of this conjecture is the affirmative result of Wojtynski for the special class of "rigged Hilbert spaces."

We have seen that the requirement that all normalized unconditional bases in a space be equivalent is very strong and that for Banach spaces, it happens only for $c_{0}, l^{1}$ and $l^{2}$. M. M. Dragilev [26] introduced the notion of quasi-equivalence of bases. Two bases $\left\{x_{n}\right\}$ and $\left\{y_{n}\right\}$ are quasiequivalent iff there are permutations $\left\{p_{n}\right\}$ and $\left\{q_{n}\right\}$ of the positive integers and nonzero numbers $a_{n}$ and $b_{n}$ such that $\left\{a_{n} x_{p_{n}}\right\}$ and $\left\{b_{n} y_{q_{n}}\right\}$ are equivalent. If $\left\{x_{n}\right\}$ and $\left\{y_{n}\right\}$ are quasi-equivalent bases in Fréchet spaces $X$ and $Y$ respectively, then $X$ and $Y$ are isomorphic. Moreover, if $X$ and $Y$ are also nuclear then the corresponding Köthe sequence spaces $\lambda\left(\left|x_{n}\right|_{m}\right)$, $\lambda\left(\left|y_{n}\right|_{m}\right)$ are identical.

Dragilev [27] has shown that for a large class of nuclear Fréchet spaces with a basis all bases in the space are quasi-equivalent. A topological vector space $E$ is a Dragilev-space if $E$ has a basis and all bases for $E$ are quasi-equivalent. ${ }^{6}$ Some outstanding problems regarding these spaces are the following:

(1) Is there a nuclear Fréchet space with a basis which is not a Dragilev space?

(2) If $E$ and $F$ are Dragilev nuclear Fréchet spaces, are the cartesian and tensor products of $E$ and $F$ Dragilev spaces?

(3) Is there a Dragilev space which is an infinite dimensional Banach space?

A noteworthy achievement on the existence of bases is due to Y. Komura and S. Koshi [61].

THEOREM 9.3. Every nuclear Fréchet vector lattice has a basis.

Y. and T. Komura [60] have exhibited a universal space $U$ for the class of nuclear Fréchet spaces, i.e., every nuclear Fréchet space $E$ is isomorphic to a closed subspace of $U$. Indeed, $U$ can be taken to be the product of countably many copies of $\lambda\left(n^{m}\right)$. Mityagin [83] has shown that $U$ is isomorphic to $C^{\infty}(-\infty, \infty)$, substantiating the conjecture of Grothendieck that this space is universal for nuclear Fréchet spaces. Now $\lambda\left(n^{m}\right)$ is a nuclear vector lattice (coordinatewise ordering) hence, so is $U$, and

\footnotetext{
${ }^{6}$ An excellent survey of results on this topic was made by Mityagin [85] who also includes some new results - mostly Russian.
} 
so every nuclear Fréchet space is a subspace of a nuclear vector lattice. Can every nuclear Fréchet space be embedded as a closed sublattice of $U$ ? This, of course, would settle the basis problem for nuclear Fréchet spaces.

Finally, we mention two recent results of A. Lazar and J. R. Retherford [63]. The notion of a Choquet simplex is needed. Let $S$ be a convex subset of a Fréchet space $E$. Passing to $E \times R(R$ the scalar field) if necessary, we may suppose that $S$ lies in a hyperplane of $E$ which misses the origin. Under this latter assumption, we say that $S$ is a Choquet simplex if the cone $C=\{a s: a \geqq 0, s \in S\}$ generated by $S$ induces a lattice ordering in $C-C$. A Choquet simplex $S$ will be called a VL-simplex iff $\bar{C}$ (the closure of $C$ ) is a cone making $\bar{C}-\bar{C}$ a topological vector lattice.

Lazar and Retherford have shown [63]

THEOREM 9.4. $A$ Fréchet space $E$ with a basis is nuclear iff every bounded set in $E$ is contained in a bounded Choquet simplex.

It is not known whether the assumption of a basis is necessary in the above theorem.

Concerning the basis problem for nuclear Fréchet spaces Lazar and Retherford [63] have shown the following equivalences.

THEOREM 9.5. If $E$ is a nuclear Fréchet space, the following are equivalent:

(i) E has a Schauder basis.

(ii) Each bounded subset of $E$ is contained in a translate of a bounded $V L$-simplex.

(iii) There is a fundamental bounded set containing 0 and contained in a translate of a bounded VL-simplex.

10. Universal bases. Let $\mathscr{B}$ be a class of bases. A basis $\left\{x_{n}\right\}$ for a Banach space is said to be universal for $\mathscr{B}$ iff for any basis $\left\{y_{k}\right\}$ in $\mathscr{B}$ there exists a subsequence $\left\{x_{n_{k}}\right\}$ such that $\left\{y_{k}\right\}$ and $\left\{x_{n_{k}}\right\}$ are equivalent, i.e., there exists an isomorphic embedding $T$ from $\left[y_{k}\right]$ into $\left[x_{n}\right]$ such that $T y_{k}=x_{n_{k}}$. If, moreover, the subsequence $\left\{x_{n_{k}}\right\}$ has the property $\sum_{n=1}^{\infty} t_{n} x_{n}$ converges implies $\sum_{k=1}^{\infty} t_{n_{k}} x_{n_{k}}$ converges, then $\left\{x_{n}\right\}$ is said to be complementably universal.

The above notions are due to Pelczynski [90] who proved the existence theorem:

THEOREM 10.1. The family $\mathscr{B}$ of all normalized bases contains a complementably universal element.

The family $\mathscr{B}$ of unconditional bases contains a complementably universal element.

Zippin [121] gives the following negative result on universal bases.

THEOREM 10.2. Let $\mathscr{B}$ be one of the following classes of normalized bases:

(a) the class of shrinking bases, 
(b) the class of all boundedly complete bases,

(c) the class of all Hilbert bases,

(d) the class of all Bessel bases,

(e) the class of all bases in Hilbert space.

Then there is no member in $\mathscr{B}$ which is universal for $\mathscr{B}$.

Remark (a) above was proved by Pelczynski [90]. Remark (b) was proved independently by Zippin [121] and Wojtaszczyk [118]. Remarks (c) and (d) are due to Zippin [121] and (e) has recently been shown by N. I. Gurarii [42].

From the above theorem and the fact that a separable Banach space has the bounded approximation property iff it is isomorphic to a complemented subspace of a Banach space with a basis comes the following corollary.

CoROllaRy 10.3. There is a separable Banach space $E$ with a complementably universal basis such that for any separable Banach space $X$ with the bounded approximation property, there are bounded linear operators $T: X \rightarrow E$ and $P: E \rightarrow X$ such that $P T=1_{X}$. In other words, any separable Banach space with the bounded approximation property is isomorphic to a complemented subspace of $E$. The space $E$ is unique up to isomorphism.

The above result is due to Johnson, Rosenthal, and Zippin and independently to Pelczynski ([51], [91]). Weaker results had been proven earlier by Pelczynski, Kadec, and Pelczynski and Wojtaszczyk ([90], [54], [94]).

Does there exist a separable Banach space $E$ such that every separable Banach space (or even every separable Banach space with the approximation property) is isomorphic to a complemented subspace of $E$ ?

The literature on bases contains two recent books. The first of these appeared in 1969 and was written by J. T. Marti [73]. It is a small book and is, as its title states, an introduction to the theory of bases. It is a good introduction going far beyond the one in Day's book [21]. Ivan Singer's book on basis theory [112] appeared in 1970. It is the first volume of a planned two volume systematic exposition of basis theory at the Banach space level. Singer's book is especially valuable as it is enhanced by many examples, and outstanding problems, and by copious historical notes. In 1963 and 1964 Ivan Singer published three papers (in Romanian) which constitute a skeleton for his two volume exposition of basis theory. These papers are not only of historical value but also are still a stimulating source of problems.

The space of holomorphic functions on the open unit disc with the topology of uniform convergence on compact sets has the powers of the complex variable $z$ as a basis. The theory of bases in Banach spaces has 
nothing to say about this familiar, simple, important basis because the above space is a nuclear Fréchet space which is not a Banach space. A systematic development of basis theory at least at the generality of Fréchet spaces is needed.

\section{REFERENCES}

1. R. D. Anderson, Hilbert space is homeomorphic to the countable infinite product of lines, Bull. Amer. Math. Soc. 72 (1966), 515-519. MR 32 \# 8298.

2. M. G. Arsove, The Paley-Wiener theorem in metric linear spaces, Pacific J. Math. 10 (1960), 365-379. MR 23 \# A2731.

3. M. G. Arsove and R. E. Edwards, Generalized bases in topological linear spaces, Studia Math. 19 (1960), 95-113. MR 22 \# 5871.

4. K. I. Babenko, On bases in Hilbert space, Dokl. Akad. Nauk SSSR 57 (1947), 427-430. (Russian) MR 9, 142.

5. G. F. Bachelis and H. P. Rosenthal, On unconditionally converging series and biorthogonal systems in a Banach space, Pacific J. Math. 37 (1971), 1-5.

6. S. Banach, Théorie des opérations linéaires, Monografie Mat., PWN, Warsaw, 1932; reprint, Chelsea, New York, 1955. MR 17, 175.

7. G. Bennett and J. B. Cooper, Weak bases in (F)- and (LF)-spaces, J. London Math. Soc. 44 (1969), 505-508. MR 39 \# 742.

8. Cz. Bessaga, On topological classification of complete linear metric spaces, Fund. Math. 56 (1964/65), 251-288. MR 31 \#2580.

9. $\mathrm{Cz}$. Bessaga and A. Pelczynski, On bases and unconditional convergence of series in Banach spaces, Studia Math. 17 (1958), 151-164. MR 22 \# 5872.

10. - A generalization of results of $R$. $C$. James concerning absolute bases in Banach spaces, Studia Math. 17 (1958), 165-174. MR 22 \# 5874.

11. - Properties of bases in spaces of type $B_{0}$, Prace Mat. 3 (1959), 123-142. (Polish) MR 23 \# A3986.

12. Cz. Bessaga, A. Pełczyński and S. Rolewicz, On diametral approximative dimension and linear homogeneity of F-spaces, Bull. Acad. Polon. Sci. Sér. Sci. Math. Astronom. Phys. 9 (1961), 677-683. MR 24 \# A2219.

13. Cz. Bessaga and A. Pełczyński, Some remarks on homeomorphisms of F-spaces, Bull. Acad. Polon. Sci. Sér. Sci. Math. Astronom. Phys. 10 (1962), 265-270. MR 25 \# 3344.

14. P. Billard, Sur les bases de Schauder dans les espaces de Banach $H^{1}$ et $A, \mathrm{C}$. R. Acad. Sci. Paris Sér. A-B 271 (1970), A36-A38.

15. Ja. M. Ceitlin, Unconditionality of a basis and partial order, Izv. Vysš. Učebn. Zaved. Matematika 1966, no. 2 (51), 98-104; English transl., Amer. Math. Soc. Transl. (2) 90 (1970), 17-25. MR 33 \# 6362; MR 41 \# 8191.

16. Z. Ciesielski, $A$ construction of bases in $C^{(1)}\left(I^{2}\right)$, Studia Math. $33(1969), 243-247$. MR 40 \# 1759.

17. T. A. Cook, Schauder decompositions and semi-reflexive spaces, Math. Ann 182 (1969), 232-235. MR 40 \# 3255.

18. W. J. Davis, D. W. Dean and Bor-Luh Lin, Bibasic sequences and norming basic sequences (to appear).

19. W. J. Davis, D. W. Dean and I. Singer, Multipliers and unconditional convergence of biorthogonal expansions, Pacific J. Math. 37 (1971), 35-39.

20. W. J. Davis and W. B. Johnson, On the existence of fundamental and total bounded biorthogonal systems in Banach spaces, Studia Math. (to appear).

21. M. M. Day, Normed linear spaces, Ergebnisse der Mathematik und ihrer Grenzgebiete Neue Folge. Heft 21. Reihe: Reelle Funktionen, Springer-Verlag, Berlin, 1958. MR 20 \# 1187.

22. - On the basis problem in normed spaces, Proc. Amer. Math. Soc. 13 (1962), 655-658. MR 25 \# 1435.

23. D. W. Dean, Schauder decompositions in $(m)$, Proc. Amer. Math. Soc. 18 (1967), 619-623. MR 36 \#664.

24. M. De Wilde, On the equivalence of weak and Schauder bases, Proc. Internat. Coll. on Nuclear Spaces and Ideals in Operator Algebras (Warsaw, 1969), Studia Math. 38 (1970), 457. 
25. J. Dieudonné, On biorthogonal systems, Michigan Math. J. 2 (1954), 7-20. MR 16, 47.

26. M. M. Dragilev, Standard form of basis for the spaces of analytic functions, Uspehi Mat. Nauk 15 (1960), no. 1 (92), 181-188. (Russian) MR 22 \#6992.

27. - On regular bases in nuclear spaces, Mat. Sb. 68 (110) (1965), 153-173; English transl., Amer. Math. Soc. Transl. (2) 93 (1970), 61-82. MR 33 \# 536; MR 42 \# 4.

28. E. Dubinsky and J. R. Retherford, Schauder bases in compatible topologies, Studia Math. 28 (1967), 221-226. MR 36 \#640.

29. - Schauder bases and Köthe sequence spaces, Trans. Amer. Math. Soc. 130 (1968), 265-280. MR 38 \#510.

30. A. S. Dynin and B. Mitjagin, Criterion for nuclearity in terms of approximative dimension, Bull. Acad. Polon. Sci. Sér. Sci. Mathl Astronom. Phys. 8 (1960), 535-540. MR 24 \# A2221.

31. P. Enflo, A Banach space with basis constant greater than 1 (to appear).

32. $\mathrm{K}$. Floret, Bases in sequentially retractive limit spaces, Proc. Internat. Coll. on Nuclear Spaces and Ideals in Operator Algebras (Warsaw, 1969), Studia Math. 38 (1970).

33. M. Fréchet, Les espaces abstraits, Gauthier-Villars, Paris, 1928.

34. R. E. Fullerton, Geometric properties of a basis in a Banach space, Proc. Internat. Congress Math. (Amsterdam, 1954), vol. 2, North-Holland, Amsterdam; Noordhoff, Groningen, 1954, p. 109.

35. - , Geometric structure of absolute basis systems in a linear topological space, Pacific J. Math. 12 (1962), 137-147. MR 26 \# 5401.

36. V. F. Gapoškin, Unconditional bases in Orlicz spaces, Uspehi Mat. Nauk 22 (1967), no. 2 (134), 113-114. (Russian) MR 34 \# 8164.

37. D. J. H. Garling, Symmetric bases of locally convex spaces, Studia Math. 30 (1968), 163-181. MR 37 \# 5670

38. D. J. H. Garling and Y. Gordon, Relations between some constants associated with finite dimensional Banach spaces, Israel J. Math. 9 (1971), 346-361.

39. B. R. Gelbaum, Notes on Banach spaces and bases, An. Acad. Brasil. Ci. 30 (1958), 29-36. MR 20 \# 5419.

40. A. Grothendieck, Produits tensoriels topologiques et espaces nucléaires, Mem. Amer.

Math. Soc. No. 16 (1955), 140 pp. MR 17, 763.

41. V. I. Gurariur, Inclination of subspaces and conditional bases in Banach spaces, Dok1

Akad. Nauk SSSR 145 (1962), 504-506 = Soviet Math. Dokl. 3 (1962), 1028-1030. MR 27 \# 2835 .

42. N. I. Gurariu, On sequences of coefficients of expansions with respect to bases in Hilbert and Banach spaces, Izv. Akad. Nauk SSSR Ser. Mat. 35 (1971), 216-223 = Math.

USSR Izv. 5 (1971), 226-232. MR 43 \# 3778.

43. L. A. Gurevič, Conic tests for bases of absolute convergence, Problems of Math. Phys. and Theory of Functions, II, "Naukova Dumka", Kiev, 1964, pp. 12-21. (Russian) MR 33 \# 7820 .

44. J. Hofler, Continuous lattice ordering by Schauder basis cones, Proc. Amer. Math. Soc. 30 (1971), 527-532.

45. R. C. James, Bases and reflexitivity of Banach spaces, Ann. of Math. (2) 52 (1950), 518-527. MR 12, 616.

46. - A non-reflexive Banach space isometric with its second conjugate space, Proc. Nat. Acad. Sci. U.S.A. 37 (1951), 174-177. MR 13, 356.

47. —— Separable conjugate spaces, Pacific J. Math. 10 (1960), 563-571. MR 22 \# 8307 .

48. W. B. Johnson, Markuschevich bases and duality theory, Trans. Amer. Math. Soc. 149 (1970), 171-177. MR 41 \# 5927.

49. - On the existence of strongly series summable Markuschevich bases in Banach spaces, Trans. Amer. Math. Soc. 157 (1971), 481-486.

50. W. P. Johnson and H. P. Rosenthal, On $w^{*}$-basic sequences and their applications to the study of Banach spaces, Studia Math. (to appear).

51. W. B. Johnson, H. P. Rosenthal and M. Zippin, On bases, finite dimensional decompositions and weaker structures in Banach spaces, Israel J. Math. 9 (1971), 488-506.

52. M. I. Kadeć, Bi-orthogonal systems and summation bases, Funkcional. Anal. i Primenen. (Trudy 5 Konf. po Funkcional' nomu Analizu i ego Primeneniju), Izdat. Akad. Nauk Azerbaìdžan. SSR, Baku, 1961, pp. 106-108. (Russian) MR 26 \# 2858.

53. - A proof of the topological equivalence of all separable infinite-dimensional Banach spaces, Funkcional. Anal. i Prilozen. 1 (1967), 61-70= Functional Anal. Appl. 1 (1967), 53-62. MR 35 \# 700.

54. - On complementably universal Banach spaces, Studia Math. 40 (1971), 85-89. 
55. M. Ц̆. Kadeć and A. Pełczyński, Basic sequences, bi-orthogonal systems and norming sets in Banach and Fréchet spaces, Studia Math. 25 (1965), 297-323. (Russian) MR 31 \# 6112.

56. N. J. Kalton, Schauder bases and reflexivity, Colloq. on Nuclear Spaces and Ideals in Operator Algebras, Studia Math. 38 (1970), 255-266.

57. ——, A barreled space without a basis, Proc. Amer. Math. Soc. 26 (1970), 465-466.

MR 42 \# 8233.

58. S. Karlin, Bases in Banach spaces, Duke Math. J. 15 (1948), 971-985. MR 10, 548.

59. Nguen Van Khue, Tests for convergence basis, Izv. Vysš. Učebn. Zaved. Matematika

1968, no. 2 (69), 68-74. (Russian) MR 36 \# 5667.

60. Y. Kōmura and T. Kōmura, Über die Einbellung der nuklearen Räume in $(s)^{A}$, Math.

Ann. $162(1965 / 66), 284-288$. MR 32 \#6190.

61. Y. Kōmura and S. Koshi, Nuclear vector lattices, Math. Ann. 163 (1966), 105-110.

MR 33 \# 553.

62. G. Köthe and $\mathrm{O}$. Toeplitz, Linear Räume mit unendlich vielen Koordinaten und Ringe

unendlicher Matrizen, J. Reine Angew. Math. 171 (1934), 193-226.

63. A. Lazar and J. R. Retherford, Nuclear spaces, Schauder bases and Choquet simplexes, Pacific J. Math. 37 (1971), 409-419.

64. M. Levin and C. W. McArthur, Order characterizations of unconditional and absolute

Schauder bases, Rev. Roumaine Math. Pures Appl. (to appear).

65. J. Lindenstrauss, On James' paper "Separable conjugate spaces", Israel J. Math. 9 (1971), 279-284.

66. J. Lindenstrauss and A. Pełczyński, Absolutely summing operators in $L_{p}$ spaces and their applications, Studia Math. 29 (1968), 275-326. MR 37 \#6743.

67. - Contributions to the theory of classical Banach spaces, J. Functional Analysis

8 (1971), 225-249.

68. J. Lindenstrauss and L. Tzafriri, On the complemented subspaces problem, Israel J.

Math. 9 (1971), 264-270.

69. - On Orlicz sequence spaces, Israel J. Math. 10 (1971), 379-390.

70. J. Lindenstrauss and $\mathrm{M}$. Zippin, Banach spaces with a unique unconditional basis, J. Functional Analysis 3 (1969), 115-125. MR 38 \# 4963.

71. E. R. Lorch, Bicontinuous linear transformations in certain vector spaces, Bull. Amer. Math. Soc. 45 (1939), 564-569. MR 1, 58.

72. A. Markuševič, Sur les basis (au sens large) dans les espaces linéaires, C. R. (Dok1.) Acad. Sci. URSS 41 (1943), 227-229. MR 6, 69.

73. J. T. Marti, Introduction to the theory of bases, Springer Tracts in Natural Philosophy, vol. 18, Springer-Verlag, Berlin and New York, 1969.

74. J. T. Marti and D. R. Sherbert, $A$ note on bases in ordered locally convex spaces, Comment. Math. Helv. 45 (1970), 299-302. MR 42 \# 5010.

75. C. W. McArthur, The weak basis theorem, Colloq. Math. 17 (1967), 71-76. MR 35 \# 7103 .

76. - The projective equicontinuous topology, Proc. Conf. on Projections, Clemson, S.C., 1967

77. - On a theorem of Orlicz and Pettis, Pacific J. Math. 22 (1967), 297-302. MR $35 \# 4702$.

78. - Convergence of monotone nets in ordered topological vector spaces, Studia Math. 34 (1970), 1-16. MR 41 \#197.

79. - In what spaces is every closed normal cone regular?, Proc. Edinburgh Math. Soc. 17 (1970), part 2, 121-125.

80. C. W. McArthur, I. Singer and M. Levin, On the cones associated with biorthogonal systems and bases in Banach spaces, Canad. J. Math. 21 (1969), 1206-1217. MR 41 \# 4207.

81. V. D. Mil'man, The geometric theory of Banach spaces, Uspehi Mat. Nauk 25 (1970), no. 3 (153), 132-138. (Russian)

82. V. D. Mil'man and Ju. B. Tumarkin, Properties of sequences in locally convex spaces, Dokl. Akad. Nauk SSSR 184 (1969), 278-281 = Soviet Math. Dokl. 10 (1969), 63-66. MR 39 \# 6045.

83. B. S. Mitjagin, Nuclearity and other properties of spaces of type $S$, Trudy Moskov. Mat. Obšč. 9 (1960), 317-328; English transl., Amer. Math. Soc. Transl. (2) 93 (1970), 45-59. MR 23 \# A2041; MR 42 \#4.

84. - - Approximative dimension and bases in nuclear spaces, Uspehi Mat. Nauk 16 (1961), no. 4 (100), 63-132 = Russian Math. Surveys 16 (1961), no. 4, 59-127. MR 27 \# 2837.

85. - - Fréchet spaces with a unique unconditional basis, Studia Math. 38 (1970), 23-34.

86. W. F. Newns, On the representation of analytic functions by infinite series, Philos. Trans. Roy. Soc. London Ser. A 245 (1953), 429-468. MR 14, 968. 
87. V. N. Nikol'skiŭ, Some questions of best approximation in a function space, Uč. Zap. Kalininsk. Pedagog. Inst. 16 (1953), 119-160. (Russian) MR 17, 175.

88. A. Pełczyński, Projections in certain Banach spaces, Studia Math. 19 (1960), 209-228. MR 23 \# A3441.

89. - Some problems on bases in Banach and Fréchet spaces, Israel J. Math. 2 (1964), 132-138. MR 30 \# 3356.

90. - - Universal bases, Studia Math. 32 (1969), 247-268. MR 39 \# 3290.

91. _ Any separable Banach space with the bounded approximation property is a complemented subspace of a Banach space with a basis, Studia Math. 40 (1971), 239-243.

92. A. Pelczyński and S. Kwapien, The main triangle projection in matrix spaces and its applications, Studia Math. 34 (1970), 43-68. MR 42 \# 5011.

93. A. Pełczyński and I. Singer, On non-equivalent bases and conditional bases in Banach spaces, Studia Math. 25 (1964/65), 5-25. MR 31 \# 3831.

94. A. Pełczyński and P. Wojtaszczyk, Banach spaces with finite dimensional expansions of identity and universal bases of finite dimensional subspaces, Studia Math. 40 (1971), 91-108.

95. J. R. Retherford, Bases, basic sequences and reflexivity of linear topological spaces, Math. Ann. 164 (1966), 280-285. MR 33 \#6351.

96. W. H. Ruckle, The infinite sum of closed subspaces of an F-space, Duke Math. J. 31 (1964), 543-554. MR 29 \#3862.

97. B. L. Sanders, Decompositions and reflexivity in Banach spaces, Proc. Amer. Math. Soc. 16 (1965), 204-208. MR 30 \# 2318.

98. S. Saxon, Basis cone base theory, Dissertation, Florida State University, Tallahassee, Fla., 1969.

99. H. H. Schaefer, Halbgeordnete lokalkonvexe Vektorräume, Math. Ann. 135 (1958), 115-141. MR 21 \# 5134.

100. - Topological vector spaces, Macmillan, New York, 1966. MR 33 \# 1689

101. J. Schauder, Zur Theorie Stetiger abbildungen in Funktionalraumen, Math. Z. 26 (1927), 47-65.

102. S. Schonefeld, Schauder bases in spaces of differentiable functions, Bull. Amer. Math. Soc. 75 (1969), 586-590. MR 39 \#6067.

103. J. H. Shapiro, On convexity and compactness in F-spaces with bases, Indiana Univ. Math. J. 21 (1972), 1073-1090.

104. J. E. Shirey and R. E. Zink, On unconditional bases in certain Banach function spaces, Studia Math. 36 (1970), 169-175.

105. I. Singer, Sur les espaces de Banach à base absolue, canoniquement équivalents à un dual d'espace de Banach, C. R. Acad. Sci. Paris 251 (1960), 620-621. MR 22 \# 5869.

106. - Sur les espaces de Banach à base absolue, canoniquement équivalents à un dual d'espace de Banach. II, C. R. Acad. Sci. Paris 251 (1960), 2456-2458. MR 22 \# 9841. 107. - Basic sequences and reflexivity of Banach spaces, Studia Math. 21 (1961/62), 351-369. MR 26 \# 4155.

108. - Bases in Banach spaces. I, Acad. R. P. Romîne Stud. Cerc. Mat. 14 (1963), 533-585. (Romanian) MR 32 \# 8121a.

109. - Bases in Banach spaces. II, Acad. R. P. Romîne Stud. Cerc. Mat. 15 (1964), 157-208. (Romanian) MR 32 \#121b.

110. - Bases in Banach spaces. III, Acad. R. P. Romîne Stud. Cerc. Mat. 15 (1964), 675-725. (Romanian) MR 32 \# 8121b.

111. - On the basis problem in topological linear spaces, Rev. Roumaine Math. Pures Appl. 10 (1965), 453-457. MR 33 \#3081.

112. - Bases in Banach spaces. I, Die Grundlehren der math. Wissenschaften, Band 154, Springer-Verlag, New York, 1970.

113. S. Smith, Cone relationships of biorthogonal systems, Pacific J. Math. 35 (1970), 787-794.

114. W. J. Stiles, On properties of subspaces of $l_{p}, 0<\mathrm{p}<1$, Trans. Amer. Math. Soc. 149 (1970), 405-415.

115. Ju. B. Tumarkin, Locally convex spaces with basis, Dokl. Akad. Nauk SSSR 195 (1970), 1278-1281 = Soviet Math. Dokl. 11 (1970), 1672-1675. MR 42 \# 6575.

116. L. J. Weill, Unconditional and shrinking bases in locally convex spaces, Pacific J. Math. 29 (1969), 467-483. MR 39 \# 7389.

117. W. Wojtyński, On conditional bases in non-nuclear Fréchet spaces, Studia Math. 35 (1970), 77-96. MR 42 \#6576.

118. P. Wojtaszczyk, On separable Banach spaces containing all separable reflexive Banach spaces, Studia Math. 37 (1971), 197-202.

119. M. Zippin, On perfectly homogeneous bases in Banach spaces, Israel J. Math. 4 (1966), 265-272. MR 35 \# 705. 
120. - A remark on bases and reflexivity in Banach spaces, Israel J. Math. 6 (1968), 74-79. MR $38 \# 4972$.

121. __ A remark on Pełczyński's paper "Universal bases", Proc. Amer. Math. Soc. 26 (1970), 294-300.

Current address: Department of Mathematics, Florida State University, Tallahassee, Florida 32306. 